\title{
Article
}

\section{Deep Learning Algorithm for Management of Diabetes Mellitus via Electrocardiogram-Based Glycated Hemoglobin (ECG-HbA1c): A Retrospective Cohort Study}

\author{
Chin-Sheng Lin ${ }^{1,+} \mathbb{D}$, Yung-Tsai Lee ${ }^{2,+}$, Wen-Hui Fang ${ }^{3}$, Yu-Sheng Lou ${ }^{4,5}$, Feng-Chih Kuo ${ }^{6} \mathbb{D}$, Chia-Cheng Lee ${ }^{7,8}$ \\ and Chin Lin $4,5,9, * \mathbb{D}$
}

Citation: Lin, C.-S.; Lee, Y.-T.; Fang, W.-H.; Lou, Y.-S.; Kuo, F.-C.; Lee, C.-C.; Lin, C. Deep Learning Algorithm for Management of Diabetes Mellitus via Electrocardiogram-Based Glycated Hemoglobin (ECG-HbA1c): A Retrospective Cohort Study. J. Pers. Med. 2021, 11, 725. https:// doi.org/10.3390/jpm11080725

Academic Editors: Giampiero Mazzaglia and Rosa Gini

Received: 30 June 2021

Accepted: 26 July 2021

Published: 27 July 2021

Publisher's Note: MDPI stays neutral with regard to jurisdictional claims in published maps and institutional affiliations.

Copyright: (c) 2021 by the authors. Licensee MDPI, Basel, Switzerland. This article is an open access article distributed under the terms and conditions of the Creative Commons Attribution (CC BY) license (https:/ / creativecommons.org/licenses/by/ $4.0 /)$.
1 Division of Cardiology, Department of Internal Medicine, Tri-Service General Hospital, National Defense Medical Center, No. 325, Section 2, Cheng-Kung Rd., Neihu, Taipei 114, Taiwan; littlelincs@gmail.com

2 Division of Cardiovascular Surgery, Cheng Hsin Rehabilitation and Medical Center, No. 45, Cheng Hsin St., Beitou, Taipei 112, Taiwan; andrewytlee.cvs@gmail.com

3 Department of Family and Community Medicine, Department of Internal Medicine, Tri-Service General Hospital, National Defense Medical Center, No. 325, Section 2, Cheng-Kung Rd., Neihu, Taipei 114, Taiwan; rumaf.fang@gmail.com

4 Graduate Institute of Life Sciences, National Defense Medical Center, No. 161, Section 6, Min-Chun E. Rd., Neihu, Taipei 114, Taiwan; chaos53438@gmail.com

5 School of Public Health, National Defense Medical Center, No. 161, Section 6, Min-Chun E. Rd., Neihu, Taipei 114, Taiwan

6 Division of Endocrinology and Metabolism, Department of Internal Medicine, Tri-Service General Hospital, National Defense Medical Center, No. 325, Section 2, Cheng-Kung Rd., Neihu, Taipei 114, Taiwan; shoummie@hotmail.com

7 Department of Medical Informatics, Tri-Service General Hospital, National Defense Medical Center, No. 325 , Section 2, Cheng-Kung Rd., Neihu, Taipei 114, Taiwan; lcgnet@gmail.com

8 Division of Colorectal Surgery, Department of Surgery, Tri-Service General Hospital, National Defense Medical Center, No. 325, Section 2, Cheng-Kung Rd., Neihu, Taipei 114, Taiwan

9 Medical Technology Education Center, School of Medicine, National Defense Medical Center, No. 161, Section 6, Min-Chun E. Rd., Neihu, Taipei 114, Taiwan

* Correspondence: xup6fup@mail.ndmctsgh.edu.tw; Tel.: +886-2-87923100 (ext. 18574); Fax: +886-2-87923147

+ These authors contributed equally to this work.

Abstract: Background: glycated hemoglobin (HbA1c) provides information on diabetes mellitus (DM) management. Electrocardiography (ECG) is a noninvasive test of cardiac activity that has been determined to be related to DM and its complications. This study developed a deep learning model (DLM) to estimate HbA1c via ECG. Methods: there were 104,823 ECGs with corresponding HbA1c or fasting glucose which were utilized to train a DLM for calculating ECG-HbA1c. Next, 1539 cases from outpatient departments and health examination centers provided 2190 ECGs for initial validation, and another 3293 cases with their first ECGs were employed to analyze its contributions to DM management. The primary analysis was used to distinguish patients with and without mild to severe DM, and the secondary analysis was to explore the predictive value of ECG-HbA1c for future complications, which included all-cause mortality, new-onset chronic kidney disease (CKD), and new-onset heart failure (HF). Results: we used a gender/age-matching strategy to train a DLM to achieve the best AUCs of 0.8255 with a sensitivity of $71.9 \%$ and specificity of $77.7 \%$ in a follow-up cohort with correlation of 0.496 and mean absolute errors of 1.230. The stratified analysis shows that DM presented in patients with fewer comorbidities was significantly more likely to be detected by ECG-HbA1c. Patients with higher ECG-HbA1c under the same Lab-HbA1c exhibited worse physical conditions. Of interest, ECG-HbA1c may contribute to the mortality (gender/age adjusted hazard ratio (HR): 1.53, 95\% conference interval (CI: 1.08-2.17), new-onset CKD (HR: 1.56, 95\% CI: 1.30-1.87), and new-onset HF (HR: 1.51, 95\% CI: 1.13-2.01) independently of Lab-HbA1c. An additional impact of ECG-HbA1c on the risk of all-cause mortality (C-index: 0.831 to $0.835, p<0.05$ ), new-onset CKD (C-index: 0.735 to $0.745, p<0.01$ ), and new-onset HF (C-index: 0.793 to $0.796, p<0.05$ ) were observed in full adjustment models. Conclusion: the ECG-HbA1c could be considered as a novel biomarker for screening DM and predicting the progression of DM and its complications. 
Keywords: artificial intelligence; electrocardiogram; deep learning; glycated hemoglobin; diabetes mellitus

\section{Introduction}

Diabetes mellitus (DM) is a critical public health issue, as this disease may affect 463 million people worldwide and will increase by $25 \%$ by 2030 and by $51 \%$ by 2045 [1] Early detection of DM improves the quality of care, contributing to fewer complications, improved survival, and higher quality of life for patients [2]. The World Health Organization guidance proposed that a glycated hemoglobin $(\mathrm{HbA1c})$ value of $6.5 \%$ is the cut-off point for diagnosing DM [3]. Although this invasive blood test may accurately identify potential DM without requiring fasting, it is limited by many conditions, causing it to be unpopular as a large-scale screening test. At present, there are various risk-scoring systems to use a set of noninvasive indicators for screening DM [4-7]. However, for these indicators, the area under the receiver operating characteristic curve (AUC) ranged from 0.72 to 0.81 in external validations [7]. Developing a more accurate noninvasive DM screening marker may reduce the burden of major complications of DM, including diabetic retinopathy (DR), diabetic neuropathy (DN), chronic kidney disease (CKD) and, particularly, cardiovascular diseases (CVDs) [8,9], which contribute to approximately 70\% of DM-related deaths and significantly increase the medical costs of diabetic patients [10]. The American Diabetes Association guidelines have therefore recommended that healthcare systems should conduct regular assessments and management of complications in diabetic patients [11]. $\mathrm{HbA1c}$ is not only useful for DM screening, but is also employed to predict DR [12], DN [13], and CKD [14]. The increase in CVD risk with rising HbA1c levels starts even without DM [15]. However, $\mathrm{HbA} 1 \mathrm{c}$ is not a regular laboratory test, which results in a large number of missing values [16], leading to difficulties when applying a screening system based on electronic medical records.

Currently, an increasing number of studies describe the use of unstructured data in the medical field [17]. For example, coronary artery calcium can be used to enhance the prediction of CVD risk [18], and another study extracted additional signals from free-text medical records on risk stratification [19]. In the past, many studies have attempted to obtain more information about the prognosis or disease diagnosis from electrocardiograms (ECG), and have successively helped to clarify the relationship between the ECG and the prognosis, but this method has not become popular because it is difficult to judge the waveform and requires other mathematical analyses. The current revolution in artificial intelligence (AI) based on deep learning models (DLMs) is a data-driven technique to learn useful features in an automated fashion [20], which is powerful for detecting myocardial infarction [21], digoxin toxicity [22], arrhythmia [23,24], hyperkalemia [25,26], left ventricular dysfunction [27,28], mitral regurgitation [29], aortic stenosis [30], and hypoglycemic events [31]. Therefore, we attempted to employ DLM to apply ECG to DM management, which may combine unstructured data to identify additional information.

DLM has also been shown to extract features that are unrecognizable to humans, such as sex and age [32]. Interestingly, patients with higher estimated ECG age, even with the same chronological age, usually have characteristics of hypertension, CAD, or a low ejection fraction [32]. We hypothesize that ECG may represent a novel biomarker for screening DM and predicting its progression. DLM may extract underlying factors when using ECG to estimate a DM progression index, such as estimating age via ECG. Because the HbA1c may be the most important factor in DM screening [3] and progression [33], we investigated the feasibility of estimating $\mathrm{HbA} 1 \mathrm{c}$ by ECG. This study aimed to train a series of DLMs using ECG to predict HbA1c, and the ECG based HbA1c (ECG-HbA1c) was decided by the DLM performance comparison. We quantified its performance on DM screening, and the underlying characteristic differences in different ECG-HbA1c under the same laboratory-based $\mathrm{HbA} 1 \mathrm{c}$ were also analyzed. Finally, we also attempted to use 
ECG-HbA1c to improve the predictive accuracy of all-cause mortality, new-onset CKD, and new-onset heart failure (HF) to validate the utility of this novel biomarker.

\section{Materials and Methods}

\subsection{Data Source and Population}

The electronic medical records of our hospital included digital ECG signals, and the records from 1 January 2012 to 31 December 2019 were available. ECGs were collected using a Philips 12-lead ECG machine (PH080A) with a 500-Hz sampling frequency and $10 \mathrm{~s}$ in each lead. The quantitative measurements and findings within the final ECG clinical reports were extracted to identify 31 diagnostic pattern classes and 8 continuous ECG measurements. The 8 ECG measurements included heart rate, PR interval, QRS duration, QT interval, correct QT interval, $\mathrm{P}$ wave axis, RS wave axis, and T wave axis. Data for these variables were $93-100 \%$ complete, and missing values were imputed using multiple imputations [34]. Patterns included abnormal T wave, atrial fibrillation, atrial flutter, atrial premature complex, complete AV block, complete left bundle branch block, complete right bundle branch block, first degree AV block, incomplete left bundle branch block, incomplete right bundle branch block, ischemia/infarction, junctional rhythm, left anterior fascicular block, left atrial enlargement, left axis deviation, left posterior fascicular block, left ventricular hypertrophy, low QRS voltage, pacemaker rhythm, prolonged QT interval, right atrial enlargement, right ventricular hypertrophy, second degree AV block, sinus bradycardia, sinus pause, sinus rhythm, sinus tachycardia, supraventricular tachycardia, ventricular premature complex, ventricular tachycardia, and Wolff-Parkinson-White syndrome. The 31 clinical diagnosis patterns were parsed from the structured findings statements on the basis of the key phrases that are standard within the Philips system. These features were used to train an extreme gradient boosting (XGB) model and elastic net, and the DLM was trained via raw ECG traces.

In this study, we used the $\mathrm{HbA1c}$ value and measured the method of ion-exchange through high performance liquid chromatography (HPLC) via running on the instrument of HLC-723G11. The ion-exchange HPLC method is certificated by the National glycohemoglobin standardization program (NGSP) as the HbA1c test with traceability to the Diabetes control and complications trial (DCCT) reference assay. The other laboratorytesting histories were collected from our electronic medical records. The diagnosis of DM was made by the following criteria [35]: (1) 6.5\% or higher on two separate HbA1c tests; (2) $126 \mathrm{mg} / \mathrm{dL}$ or higher on two separate GLU tests; (3) $200 \mathrm{mg} / \mathrm{dL}$ or higher after two hours in oral glucose tolerance test. Patients meeting any of the above criteria before the date of ECG were identified. Moreover, patients with a HbA1c of $\geq 8.0 \%$ were defined as a severe DM at the date of ECG. In this study, we classified patients with prediabetes as a non-DM group in following analyses, which were defined with the following criteria: (1) $5.7 \%$ or higher on two separate $\mathrm{HbA1c}$ tests; (2) $100 \mathrm{mg} / \mathrm{dL}$ or higher on two separate GLU tests; (3) $140 \mathrm{mg} / \mathrm{dL}$ or higher after two hours in an oral glucose tolerance test.

Figure 1 shows the generation of study cohorts. There were 23,195 patients visiting the outpatient department (OPD) in the study period with more than 1 ECG and $\mathrm{HbA} 1 \mathrm{c}$ pair within 30 days. Among them, there were 5084 patients visiting the OPD earlier than 1 January 2015, and 2098 patients visiting the OPD from 1 January 2015 to 31 December 2015. Only patients that had an ECG and $>1 \mathrm{HbA} 1 \mathrm{c}$ measurement within 3 days were included, leading to the remaining 3293 patients and 1539 patients before 1 January 2015 and between 1 January 2015 and 31 December 2015, respectively. There were 2190 ECGs from 1539 people in the validation cohort for initially validating the DLMs. As for the 3293 patients, only the earliest ECG was applied to generate a follow-up cohort with 3293 ECGs, which were used for the accuracy test of DLM and the evaluation of the meaning of prediction error. In the validation cohort, $752(34.3 \%)$ patients had no history of DM, $454(20.7 \%)$ patients had the history of prediabetes with the duration of $1.19 \pm 1.83$ years, and $984(44.9 \%)$ patients had the history of DM with the duration of $4.84 \pm 3.83$ years, while the followup cohort consisted of $816(24.8 \%)$ patients without DM, $528(16.0 \%)$ patients with the 
history of prediabetes and the duration of $1.75 \pm 2.36$ years, and 1949 (59.2\%) patients with the history of DM and the duration of $4.69 \pm 3.77$ years. Based on this sample size for following the outcomes with hypothetical incidences of $1 \% / 5 \%$, the statistical powers achieved $65.6 \% />99.9 \%$ using the following settings: a significance level of 0.05 , a ratio of two groups was equal to 1 , and a minimum detectable relative risk of 2 . We selected the earliest data as the follow-up cohort for maximizing the following time of DM related outcomes. There was no overlap among the cohorts.

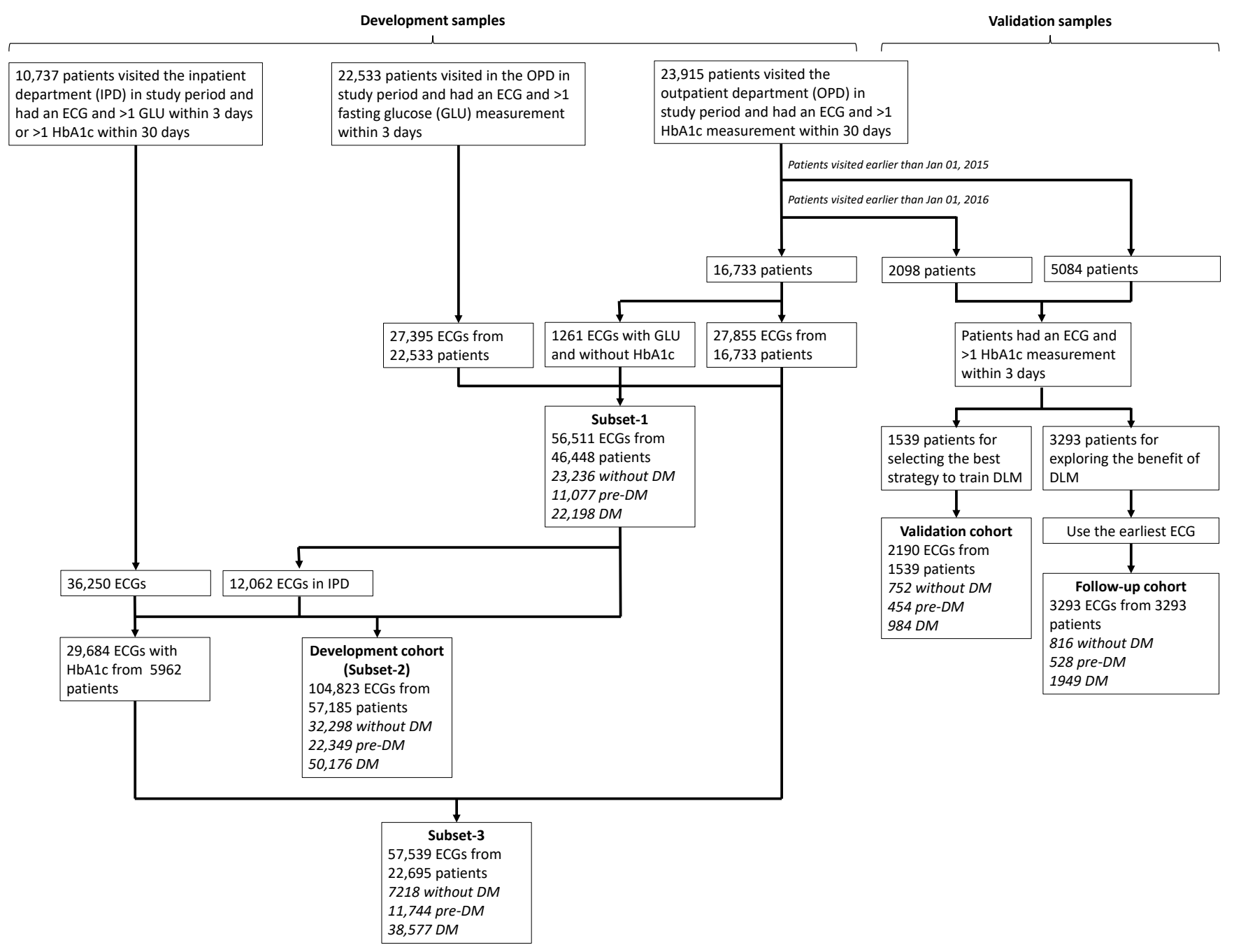

Figure 1. The summary of study design in this study. The process of development, validation, and follow-up cohorts with each electrocardiogram (ECG) labeling of HbA1c was indicated. The patients in validation and follow-up cohorts were totally different from development cohort. The development cohort included three subsets (subset-1: outpatient department samples; subset-2: full samples; subset-3: samples with corresponding HbA1c). Abbreviations: DM, diabetes mellitus.

We used a series of methods to collect more samples for developing DLMs. The remaining 16,733 patients first visited after 1 January 2016 had 27,855 ECGs with corresponding $\mathrm{HbA1c}$ in the OPD. For further increasing the data volume, we included ECGs without corresponding $\mathrm{HbA1c}$ but with corresponding fasting glucose (GLU) within 3 days. A previous study developed an equation for estimating average GLU as follows: $28.7 \times \mathrm{HbA} 1 \mathrm{C}-46.7$ [36], and we used the inverse function to calculate the estimated $\mathrm{HbA1c}$. This method increased to 1261 ECGs with corresponding GLU and without $\mathrm{HbA1c}$ from 16,733 patients with more than 1 ECG and GLU pair. Further, 27,395 ECGs with estimated $\mathrm{HbA} 1 \mathrm{c}$ from the other 22,533 patients in study period were collected. Therefore, a total of 56,511 ECGs from 46,448 patients were used to construct subset-1 with only OPD data included. To further augment the development samples, 10,737 patients who visited 
the inpatient department (IPD) were included in the study period, with 36,250 ECGs and corresponding $\mathrm{HbA} 1 \mathrm{c}$ within 30 days or estimated $\mathrm{HbA} 1 \mathrm{c}$ within 3 days. There were 12,062 IPD ECGs from the 46,448 patients in subset- 1 using the same criteria, and there were 104,823 ECGs from 57,185 patients in the subset-2. We further excluded the ECGs without corresponding $\mathrm{HbA} 1 \mathrm{c}$ to construct subset- 3 with the remaining 57,539 ECGs from 22,695 patients. We defined the subset-2 as the major development cohort. There were $32,298(30.8 \%)$ patients without DM, 22,695 (21.3\%) patients with the history of prediabetes and the duration of $0.88 \pm 1.91$ years, and 50,176 (47.9\%) patients with history of DM and the duration of $4.33 \pm 4.07$ years.

\subsection{Observational Variables}

In addition to glucose profile, we also collected the relevant blood laboratory values in the OPD, including electrolytes, liver and renal function profiles, albumin (Alb), c-reactive protein (CRP), complete blood cell count, and lipid profiles. The nearest laboratory test was obtained within 3 days before and after enrollment. The missing data were imputed using multiple imputations in multivariable analysis [34].

The complications of this study in the follow-up cohort were all-cause mortality, newonset CKD, and new-onset HF. For the mortality data, the survival time was calculated with reference to the date of ECG. Patient status (dead/alive) was defined through electronic medical records, which were updated by each hospital activity. Moreover, data for alive visits were censored at the patient's last known hospital alive encounter to limit bias from incomplete records. The end of follow-up in this study was 31 December 2019. Patients without revisits to our hospital were excluded, and there were 3288 (99.8\%) at risk samples for mortality analysis.

The new-onset CKD event was defined as at least 2 records of estimated glomerular filtration rate $(\mathrm{eGFR}) \leq 60 \mathrm{~mL} / \mathrm{min}$ or markers of kidney damage (albumin to creatinine ratio $\geq 30 \mathrm{mg} / \mathrm{g}$ or positive urine strip test) after the index date. Patients meeting any of the above criteria before the date of ECG were excluded and defined as having CKD history, and the number of at risk patients was 2426 . The HF was defined by the quantitative ejection fraction recorded at the acquisition in the Philips image system ${ }^{\circledR}$. The ejection fraction is routinely acquired by experienced cardiologist or technicians using a standardized method. An ejection fraction of $\leq 35 \%$ was defined as HF in this study, and the history of HF and at risk patients followed the above rules. There were 3031 at risk patients to follow up on the new-onset HF.

The other disease histories were based on the corresponding International Classification of Diseases, Ninth Revision and Tenth Revision (ICD-9 and ICD-10, respectively) as follows: hypertension (HTN, ICD-9 codes 401.x to 404.x and ICD-10 codes I10.x to I16.x), hyperlipidemia (HLP, ICD-9 codes 272.x and ICD-10 codes E78.x), stroke (STK, ICD-9 codes 430.x to 438. $x$ and ICD-10 codes I60. $x$ to I63.x), coronary artery disease (CAD, ICD-9 codes 410.x to 414.x, and 429.2, and ICD-10 codes I20.x to I25.x), atrial fibrillation (AF, ICD-9 codes 427.31 and ICD-10 codes I48.x), and chronic obstructive pulmonary disease (COPD, ICD-9 codes 490. $x$ to 496.x and ICD-10 codes J44.9).

\subsection{Implementation of the Deep Learning Model}

The DLM architecture with an attention mechanism was used to estimate $\mathrm{HbA} 1 \mathrm{c}$, which was based on our previous study [21,22,26,37]. Figure 2A shows the architecture of our DLM. Each ECG was recorded as a standard 12 leads consisting of 5000 number sequences, and a $5000 \times 12$ matrix was generated based on these sequences. An input format of this architecture is a $4096 \times 12$ matrix. We randomly cropped a length of 4096'sequences as input during the training process. For the inference stage, 2 overlapping lengths of 4096 sequences at the start and the end were used to generate predictions that were averaged as the final prediction. 
A
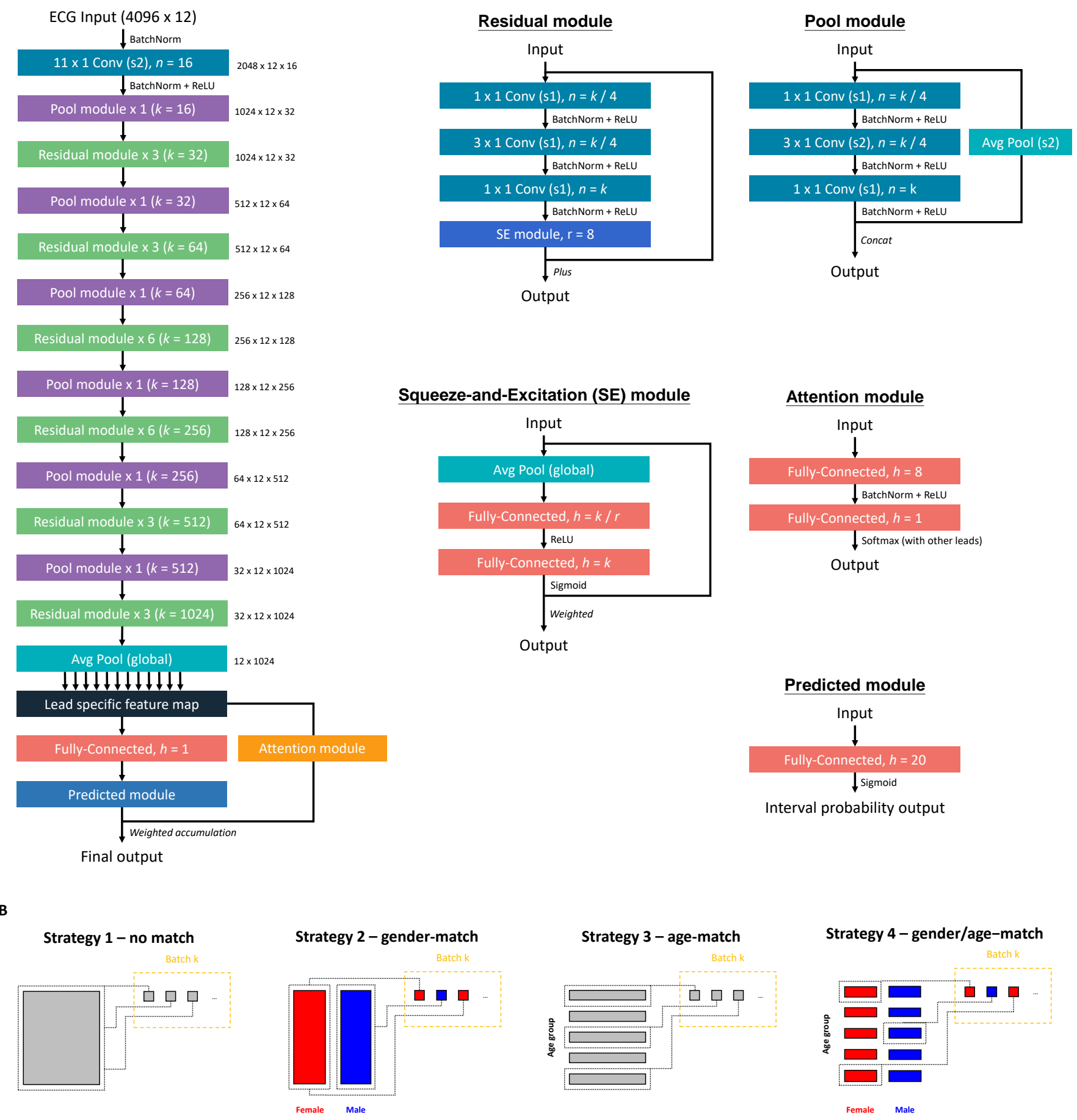

Final output

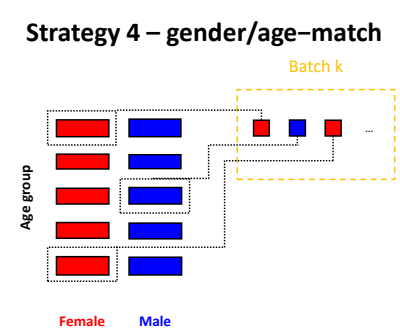

Figure 2. The implementation of our deep learning model. (A) The model architectures of the deep learning model for analyzing ECG. (B) Four training strategies were based on different sampling processes. The matching strategy was to split the sample to multiple blocks based on different conditions. The batch samples were sampled from each block with the same probability.

We defined a "residual module" as a neural combination with a constant $k$, as follows: (1) a $1 \times 1$ convolution layer with $k / 4$ filters to reduce the dimensions of the data, (2) a batch normalization layer to normalization, (3) a rectified linear unit (ReLU) layer for non-linearization, (4) a $3 \times 1$ convolution layer with $k / 4$ filters to extract features, (5) a batch normalization layer for normalization, (6) a ReLU layer for non-linearization, and 
(6) a $3 \times 1$ convolution layer with $4 \mathrm{~K}$ filters to extract features, (7) a $1 \times 1$ convolution layer with $k$ filters to restore feature shape, (8) a batch normalization layer for normalization, (9) a ReLU layer for non-linearization, and (10) a squeeze-and-excitation (SE) module for weighting features. The SE module was defined as follows: (1) an average global pooling layer, (2) a fully-connected layer with $k / r$ neurons, and (3) a fully-connected layer with $k$ neurons. The constant $r$ was set at 8 in all experiments. The residual module was ended by a shortcut connection, resulting in direct connections of each layer with all subsequent layers.

The residual module cannot be concatenated when the size of feature maps changes. Thus, a "pool module" was used to concatenate each residual module for down-sampling in our architecture. This module included similar concatenated layers with residual modules, but the stride of the $3 \times 1$ convolution layer was changed to $2 \times 1$. An average pooling layer with a $2 \times 1$ kernel size and stride was used for down-sampling. We used the concatenated function to integrate them.

The input data were passed through a batch normalization layer, followed by a $11 \times 1$ convolution layer with $2 \times 1$ stride and 16 filters, another batch normalization layer, a ReLU layer, and a pool module. Next, the data were passed through a series of residual modules and pool modules, resulting in a $32 \times 12 \times 1024$ array. A global pooling layer was followed by the last residual module. We divided it into 12 lead-specific feature maps with 1024 features. These feature maps were passed through a fully-connected layer with 1 neuron to generate the lead specific predictions. We designed an attention mechanism based on a hierarchical attention network to concatenate these blocks, increasing the interpretive power of DLM. The attention module was comprised of a fully connected layer with 8 neurons, followed by a batch normalization layer, a ReLU layer, and a fullyconnected layer with 1 neuron to generate the weights of each lead. Attention scores were calculated for each ECG lead and then integrated for standardization by the last linear output layer. The standardized attention scores were used to weight the 12 ECG lead outputs by simple multiplication. The 12 weighted outputs were summed and passed through a predicted module to give the final prediction value.

To increase the nonlinear adaptability and reduce monotonously linear predicted functions of outputs, we used the category-wise label encoding technology to code the $\mathrm{HbA} 1 \mathrm{c}$ concentration. The range of $\mathrm{HbA} 1 \mathrm{c}$ concentration was defined from $4.0 \%$ to $10.0 \%$. We designed a 20 sigmoid output by an interval of 0.3 . For example, the minimal HbA1c concentration of less than $4.0 \%$ is coded as $(0,0,0,0,0,0,0,0,0,0,0,0,0,0,0,0,0,0,0,0)$, the $\mathrm{HbA} 1 \mathrm{c}$ concentration of $6.5 \%$ is coded as $(1,1,1,1,1,1,1,1,1,0,0,0,0,0,0,0,0,0,0,0)$, the $\mathrm{HbA} 1 \mathrm{c}$ concentration of $8.0 \%$ is coded as $(1,1,1,1,1,1,1,1,1,1,1,1,1,1,0,0,0$, $0,0,0)$, and so on. The loss function is cross-entropy in these sigmoid outputs, and our network was trained to minimize the cross entropy loss. The final prediction was the sum of these values multiplied by 0.3 plus 4.0 . For example, a prediction vector given by DLM is $(1,1,1,1,1,1,1,1,0.9,0.8,0.7,0,0,0,0,0,0,0,0)$, which corresponds to an ECG-HbA1c concentration of $7.12 \%$.

An oversampling process was implemented to ensure that rare cases with extreme $\mathrm{HbA1c}$ values were adequately recognized, which was based on weights computed on the prevalence of 20 equidistant intervals in the development cohort. In our study, the distribution of $\mathrm{HbA} 1 \mathrm{c}$ was not uniform, therefore the ECGs with rare values were copies of existing samples at random to increase the number of observations. This ideally gives us a sufficient number of samples to play with [38,39]. However, we explored multiple oversampling strategies to maximize the model's performance because ECG was related to gender and age [32]. Figure 2B shows the summary of four training strategies. The first strategy was the oversampling process based on the reciprocals of prevalence of 20 equidistant intervals in each batch (no match). The second strategy was to ensure a balanced gender distribution in each batch (gender-match). The third strategy was to additionally consider the weight of age, which was also computed on the prevalence of 20 equidistant intervals in the development cohort (age-match). The fourth strategy was matching both gender and age (gender/age-match). We compared the matching effects 
of the 4 trained DLMs using a full-scale development cohort. A sensitivity analysis using only ECGs from OPD (subset-1) and ECGs with corresponding HbA1c (subset-3) was conducted.

We trained these DLMs with a 32 batch size and used an initial learning rate of 0.001 using an Adam optimizer with standard parameters $\left(\beta_{1}=0.9\right.$ and $\left.\beta_{2}=0.999\right)$. The learning rate was decayed by a factor of 10 each time the loss of the validation cohort plateaued after an epoch. To prevent the networks from overfitting, early stopping was performed by saving the network after every epoch and choosing the saved DLMs with the lowest loss on the validation cohort. The only regularization method for avoiding overfitting was the L2 regularization with a coefficient of $10^{-4}$ in this study.

Abbreviations: OPD, outpatient department; HEC, health examination center; IPD, in-patient department; EMR, emergency room; BMI, body mass index; SBP, systolic blood pressure; DBP, diastolic blood pressure; DM, diabetes mellitus; HTN, hypertension; HLP, hyperlipidemia; CKD, chronic kidney disease; STK, stroke, CAD, coronary artery disease; HF, heart failure; $\mathrm{AF}$, atrial fibrillation; $\mathrm{COPD}$, chronic obstructive pulmonary disease; $\mathrm{HbA1c}$, glycated hemoglobin; GLU, glucose AC; eGFR, estimated glomerular filtration rate; $\mathrm{BUN}$, blood urea nitrogen; $\mathrm{Na}$, sodium; $\mathrm{K}$, potassium; $\mathrm{Cl}$, chloride; $\mathrm{Ca}$, total calcium; $\mathrm{Mg}$, magnesium; Alb, albumin; CRP, C-reactive protein; WBC, white blood cell count; PLT, platelet; Hb: hemoglobin; AST, aspartate aminotransferase; ALT, alanine aminotransferase; TG, triglyceride; TC, total cholesterol; LDL, low density lipoprotein cholesterol; HDL, high density lipoprotein cholesterol.

\subsection{Statistical Analysis and Model Performance Assessment}

Patient characteristics are presented as means and standard deviations, numbers of patients, or percentages where appropriate and were compared using either analysis of variance, Student's t-test, or Chi-square test, as appropriate. All statistical analyses were completed in $\mathrm{R}$ version 3.4.4. The significance level was set as $p<0.05$. We provided a series of DLMs with training via different strategies, and the optimal DLM was selected based on the highest AUC for detecting DM in the validation cohort. Moreover, the results of XGB model and elastic net were presented, which provided corresponding variable important rankings to explore the relationship between explainable features and HbA1c.

The primary analysis was to explore the diagnostic value on DM and severe DM in the follow-up cohort. The AUC, sensitivity (recall), specificity, precision, and F-measure are presented. Moreover, confusion scatter plots with mean absolute error (MAE) were used to compare actual HbA1c and ECG-HbA1c. The stratified analysis was also conducted. The secondary analysis was to explain the estimation residual between laboratory-based and ECG-based HbA1c. We explored the difference in characteristics in each ECG-HbA1c group sharing the same Lab-HbA1c. Linear regression or logistic regression was used for statistical testing where appropriate. Finally, we used univariable and multivariable Cox proportional hazard models to analyze the relationship between baseline characteristics and outcomes of interest. Hazard ratios (HRs) and 95\% conference intervals (95\% CIs) were used for comparison. A series of integration models were evaluated using the C-index as global performance to explore the additional contributions of ECG-HbA1c.

\section{Results}

Table 1 shows patient characteristics in the development, validation, and follow-up cohorts. Almost all characteristics were different among these three cohorts, which were grouped by date. This might reduce the generalizability of DLM if it was learned via spurious relationships. The number of mortalities was 61 (1.9\%) during a median followup period of 4.5 years, and the incidence of new-onset CKD and HF was $8.3 \%$ (201) and $2.8 \%(86)$, respectively.

We next explored a suitable DLM training strategy for subsequent analysis. Figure $3 \mathrm{~A}$ shows that the $\mathrm{HbA1c}$ predicted by DLM with gender/age-match provided the highest AUC of 0.855 (95\% CI: $0.840-0.871)$ for detecting DM, which was the most highly corre- 
lated with laboratory-based $\mathrm{HbA} 1 \mathrm{c}(r=0.557,95 \% \mathrm{CI}$ : 0.531-0.582). Figure 3B shows the performances of the best DLM, XGB model, and elastic net for detecting DM and severe DM in the follow-up cohort. The AUCs of DLM with gender/age-match, XGB model, and elastic net on DM was $0.8255,0.7573$, and 0.7226 in the follow-up cohort, respectively. Our ECG-HbA1c shows a sensitivity of $71.9 \%$ and specificity of $77.7 \%$ in the detection of DM. For patients with DM, we observed an AUC of 0.6550 using DLM for detecting severe DM in the follow-up cohort, which was better than the XGB model (0.5961) and elastic net (0.5884). Therefore, the ECG-HbA1c was defined as the estimation result of DLM with a gender/age-match. The scatter plot with $\mathrm{Lab}-\mathrm{HbA} 1 \mathrm{c}$ versus ECG-HbA1c is presented in Figure 3C. The mean absolute errors of Lab-HbA1c and ECG-HbA1c in the follow-up cohort was 1.238 with correlations of 0.493 . Figure 3D shows the most important role of heart rate in the prediction of $\mathrm{HbA1c}$ in the XGB model, while a corrected QT interval, QT interval, followed by an RS wave axis played vital roles in the elastic net.

Table 1. Patient characteristics and laboratory results in development, validation, and follow-up cohorts.

\begin{tabular}{|c|c|c|c|c|}
\hline & $\begin{array}{l}\text { Development Cohort } \\
(N / n=57,185 / 104,823)\end{array}$ & $\begin{array}{l}\text { Validation Cohort } \\
(N / n=1539 / 2190)\end{array}$ & $\begin{array}{l}\text { Follow-Up Cohort } \\
(N / n=3293 / 3293)\end{array}$ & $p$-Value \\
\hline Location & & & & $<0.001$ \\
\hline OPD/HEC & $56,511(53.9 \%)$ & $2190(100.0 \%)$ & $3293(100.0 \%)$ & \\
\hline IPD/EMR & $48,312(46.1 \%)$ & $0(0.0 \%)$ & $0(0.0 \%)$ & \\
\hline Gender (Male) & $59182(56.5 \%)$ & $1124(51.3 \%)$ & $1746(53.0 \%)$ & $<0.001$ \\
\hline Age (years) & $60.9 \pm 17.1$ & $56.0 \pm 14.8$ & $58.8 \pm 15.0$ & $<0.001$ \\
\hline $\mathrm{BMI}\left(\mathrm{kg} / \mathrm{m}^{2}\right)$ & $25.2 \pm 6.0$ & $24.8 \pm 3.9$ & $25.5 \pm 4.2$ & $<0.001$ \\
\hline $\mathrm{SBP}(\mathrm{mmHg})$ & $136.0 \pm 27.9$ & $130.3 \pm 25.0$ & $134.4 \pm 26.7$ & $<0.001$ \\
\hline $\mathrm{DBP}(\mathrm{mmHg})$ & $79.3 \pm 17.1$ & $79.3 \pm 14.8$ & $79.5 \pm 15.4$ & 0.752 \\
\hline \multicolumn{5}{|l|}{ Disease history } \\
\hline $\mathrm{DM}$ & $50,176(47.9 \%)$ & $984(44.9 \%)$ & $1949(59.2 \%)$ & $<0.001$ \\
\hline HTN & $42,116(40.2 \%)$ & $846(38.6 \%)$ & $1773(53.8 \%)$ & $<0.001$ \\
\hline HLP & $41,117(39.2 \%)$ & $880(40.2 \%)$ & $1767(53.7 \%)$ & $<0.001$ \\
\hline CKD & $34,246(32.7 \%)$ & $438(20.0 \%)$ & $862(26.2 \%)$ & $<0.001$ \\
\hline STK & $13,893(13.3 \%)$ & $216(9.9 \%)$ & $430(13.1 \%)$ & $<0.001$ \\
\hline CAD & $24,474(23.3 \%)$ & $508(23.2 \%)$ & $1059(32.2 \%)$ & $<0.001$ \\
\hline $\mathrm{HF}$ & $6693(6.4 \%)$ & $119(5.4 \%)$ & $256(7.8 \%)$ & 0.001 \\
\hline $\mathrm{AF}$ & $4983(4.8 \%)$ & $70(3.2 \%)$ & $125(3.8 \%)$ & $<0.001$ \\
\hline COPD & $13,555(12.9 \%)$ & $239(10.9 \%)$ & $595(18.1 \%)$ & $<0.001$ \\
\hline \multicolumn{5}{|l|}{ Laboratory test } \\
\hline HbA1c (\%) & $7.0 \pm 1.8$ & $6.3 \pm 1.4$ & $6.6 \pm 1.6$ & $<0.001$ \\
\hline GLU (mg/dL) & $119.1 \pm 49.3$ & $115.5 \pm 43.9$ & $123.2 \pm 49.1$ & $<0.001$ \\
\hline eGFR (mL/min) & $81.6 \pm 36.2$ & $89.2 \pm 27.1$ & $84.5 \pm 30.3$ & $<0.001$ \\
\hline BUN (mg/dL) & $22.1 \pm 19.6$ & $16.5 \pm 9.7$ & $18.8 \pm 13.7$ & $<0.001$ \\
\hline $\mathrm{Na}(\mathrm{mmol} / \mathrm{L})$ & $137.8 \pm 4.8$ & $139.0 \pm 3.8$ & $138.5 \pm 4.2$ & $<0.001$ \\
\hline $\mathrm{K}(\mathrm{mmol} / \mathrm{L})$ & $4.0 \pm 0.5$ & $4.1 \pm 0.4$ & $4.1 \pm 0.5$ & $<0.001$ \\
\hline $\mathrm{Cl}(\mathrm{mEq} / \mathrm{L})$ & $103.3 \pm 5.0$ & $103.8 \pm 3.7$ & $103.5 \pm 4.4$ & $<0.001$ \\
\hline $\mathrm{Ca}(\mathrm{mg} / \mathrm{dL})$ & $9.0 \pm 0.7$ & $9.2 \pm 0.5$ & $9.1 \pm 0.6$ & $<0.001$ \\
\hline $\mathrm{Mg}(\mathrm{meq} / \mathrm{L})$ & $2.1 \pm 0.3$ & $2.1 \pm 0.2$ & $2.1 \pm 0.3$ & 0.122 \\
\hline Alb (g/dL) & $3.9 \pm 0.7$ & $4.2 \pm 0.5$ & $4.1 \pm 0.5$ & $<0.001$ \\
\hline $\mathrm{CRP}(\mathrm{mg} / \mathrm{L})$ & $2.8 \pm 5.5$ & $1.4 \pm 3.3$ & $1.8 \pm 3.9$ & $<0.001$ \\
\hline $\operatorname{WBC}\left(10^{3} / \mu \mathrm{L}\right)$ & $8.3 \pm 5.1$ & $7.0 \pm 4.7$ & $7.4 \pm 3.2$ & $<0.001$ \\
\hline $\operatorname{PLT}\left(10^{3} / \mu \mathrm{L}\right)$ & $235.4 \pm 81.3$ & $237.3 \pm 68.1$ & $234.9 \pm 71.7$ & 0.504 \\
\hline $\mathrm{Hb}(\mathrm{mg} / \mathrm{dL})$ & $13.1 \pm 2.3$ & $13.6 \pm 1.9$ & $13.5 \pm 2.1$ & $<0.001$ \\
\hline AST (U/L) & $35.9 \pm 119.8$ & $22.3 \pm 15.8$ & $25.0 \pm 21.8$ & $<0.001$ \\
\hline ALT (U/L) & $31.8 \pm 103.2$ & $22.5 \pm 17.0$ & $25.0 \pm 25.6$ & $<0.001$ \\
\hline $\mathrm{TG}(\mathrm{mg} / \mathrm{dL})$ & $136.6 \pm 131.0$ & $137.5 \pm 104.7$ & $145.7 \pm 157.9$ & $<0.001$ \\
\hline $\mathrm{TC}(\mathrm{mg} / \mathrm{dL})$ & $172.0 \pm 48.8$ & $179.4 \pm 38.3$ & $178.5 \pm 41.7$ & $<0.001$ \\
\hline $\mathrm{LDL}(\mathrm{mg} / \mathrm{dL})$ & $102.9 \pm 37.5$ & $108.2 \pm 33.4$ & $107.4 \pm 34.9$ & $<0.001$ \\
\hline $\mathrm{HDL}(\mathrm{mg} / \mathrm{dL})$ & $46.7 \pm 15.2$ & $49.4 \pm 13.6$ & $48.5 \pm 14.0$ & $<0.001$ \\
\hline
\end{tabular}


Figure 4 shows that DLM performance was strong across all conditions to detect DM. The strengths of association, albeit widely inconsistent in different conditions for DM detection, were much higher for female and younger patients with fewer co-morbidities (HTN, HLP, and STK) and low BMI. The DLM exhibits higher AUCs with higher specificities in health patients, which indicated that patients with complex co-morbidities were more likely to be recognized as DM by ECG-HbA1c. This implied that patients with normal ECG-HbA1c but abnormal Lab-HbA1c (false negative) were young and healthy, while patients with abnormal ECG-HbA1c but normal Lab-HbA1c (false positive) were elderly with co-morbidities. Intriguingly, these patient characteristics had no impacts on the performance difference of severe DM detection.

A Correlation analysis

0.8
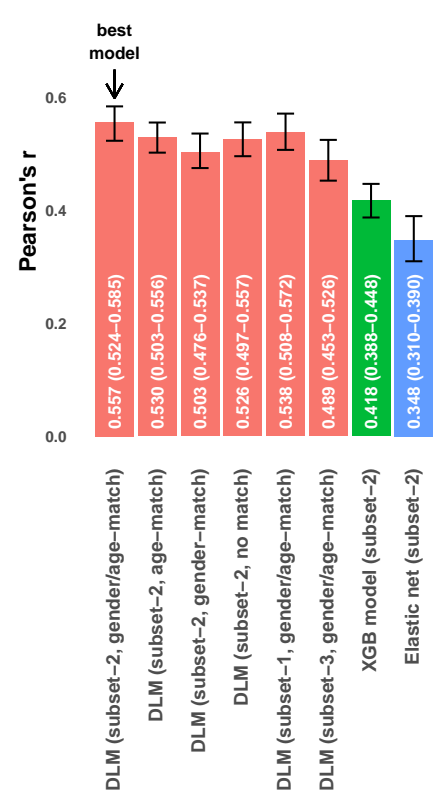

C

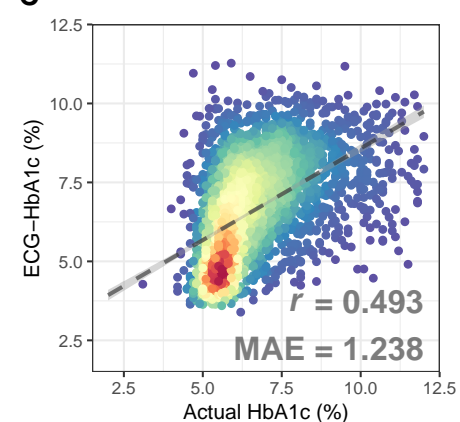

ROC curve analysis
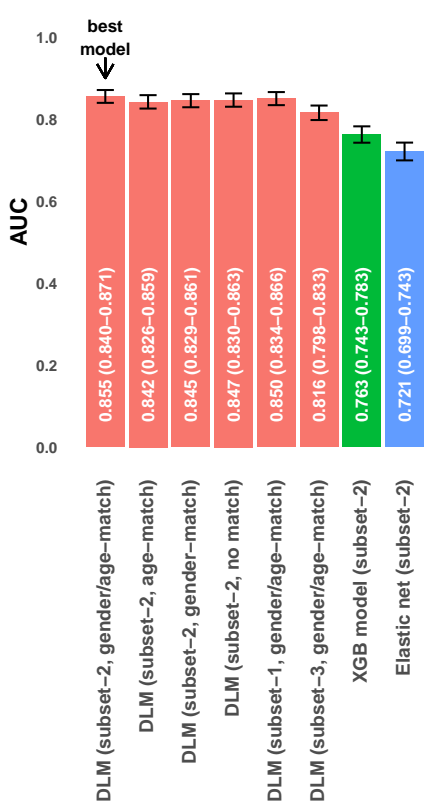

D

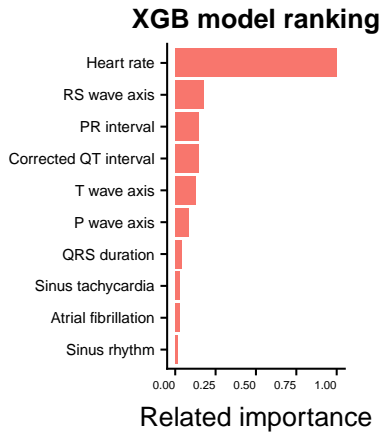

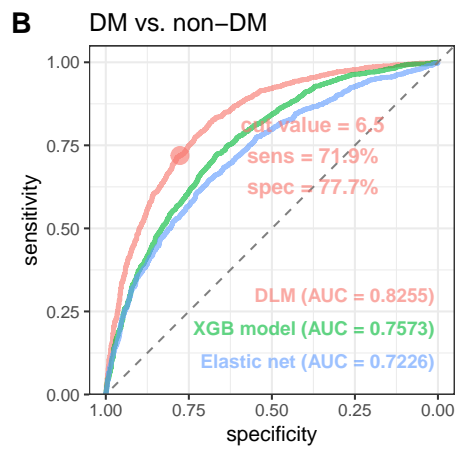

B DM vs. non-DM

severe DM vs. mild DM
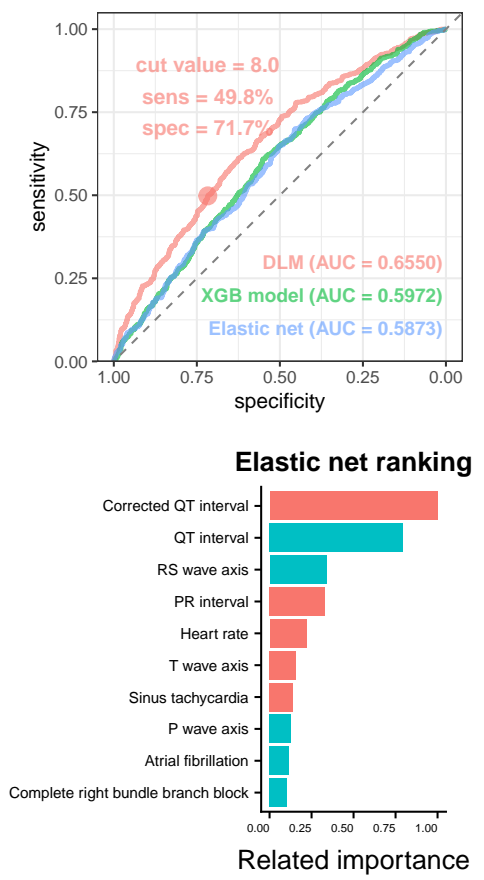

Figure 3. Analysis of deep learning model and traditional machine learning models. (A) The performance comparison of deep learning model (DLM) trained by 6 different weighting strategies in the validation cohort. The DLM ( ... ) were made by predictions of deep learning models using different strategies. The XGB model and Elastic net demonstrated the corresponding predictions. The gender/age-matching strategy provides the highest correlation between estimated $\mathrm{HbA1c}$ and actual HbA1c. (B) Performance comparison for detecting DM and severe DM in the follow-up cohort. ROC curves were created from predictions of the deep learning model trained using a gender/age-matching strategy. Moreover, the performance of XGB model and elastic net were also presented. (C) Scatter plot between DLM predictions and actual $\mathrm{HbA1c}$ in the follow-up cohort. The $x$-axis indicates the true $\mathrm{HbA1c}$ from laboratory tests. The $y$-axis presents the predicted $\mathrm{HbA} 1 \mathrm{c}$ from the deep learning model trained using a gender/age-matching strategy. Red points represent the highest density, followed by yellow, green, light blue, and dark blue. (D) Related feature importance ranking in XGB model (information gain) and elastic net (standard coefficient). There are only the top $10 \mathrm{important}$ variables in each model, and the blue color demonstrates the negative relationship between variables and actual $\mathrm{HbA1c}$. 


\begin{tabular}{|c|c|c|c|c|c|c|c|c|}
\hline \multirow{4}{*}{$\begin{array}{l}\text { All patients } \\
\text { Gender }\end{array}$} & \multicolumn{5}{|c|}{ DM vs. non-DM } & \multicolumn{3}{|c|}{ severe DM vs. mild DM } \\
\hline & Spec (\%) & Sens (\%) & & AUC $(95 \% \mathrm{Cl})$ & Spec (\%) & Sens (\%) & & AUC $(95 \% \mathrm{Cl})$ \\
\hline & $77.7 \%(1044 / 1344)$ & $71.9 \%(1402 / 1949)$ & н & $0.826(0.811-0.840)$ & $71.7 \%(1019 / 1421)$ & $49.8 \%(263 / 528)$ & 且 & $0.655(0.628-0.682)$ \\
\hline & & & & $p=0.0001$ & & & & $p=0.0118$ \\
\hline Male & $72.1 \%(511 / 709)$ & $72.4 \%(751 / 1037)$ & в & $0.798(0.777-0.819)$ & $70.3 \%(536 / 762)$ & $48.7 \%(134 / 275)$ & 日 & $0.622(0.583-0.661)$ \\
\hline Female & $83.9 \%(533 / 635)$ & $71.4 \%(651 / 912)$ & н & $0.856(0.837-0.875)$ & $73.3 \%(483 / 659)$ & $51.0 \%(129 / 253)$ & 日 & $0.691(0.654-0.728)$ \\
\hline Age group & & & & $p=0.0014$ & & & & $p=0.3374$ \\
\hline Age $<65$ & $80.3 \%(943 / 1175)$ & $67.6 \%(652 / 964)$ & н & $0.821(0.803-0.838)$ & $71.5 \%(481 / 673)$ & $51.9 \%(151 / 291)$ & 日 & $0.669(0.632-0.705)$ \\
\hline Age $>65$ & $59.8 \%(101 / 169)$ & $76.1 \%(750 / 985)$ & 日 & $0.750(0.711-0.790)$ & $71.9 \%(538 / 748)$ & $47.3 \%(112 / 237)$ & ت⿴囗十 & $0.642(0.603-0.682)$ \\
\hline BMI group & & & & $p<0.0001$ & & & & $p=0.7452$ \\
\hline $\mathrm{BMI}<24$ & $88.7 \%(575 / 648)$ & $67.3 \%(431 / 640)$ & H & $0.870(0.850-0.889)$ & $75.3 \%(351 / 466)$ & $44.8 \%(78 / 174)$ & 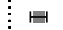 & $0.663(0.617-0.709)$ \\
\hline $\mathrm{BMI}>24$ & $67.4 \%(469 / 696)$ & $74.2 \%(971 / 1309)$ & 由 & $0.777(0.755-0.798)$ & $69.9 \%(668 / 955)$ & $52.3 \%(185 / 354)$ & 日 & $0.653(0.620-0.687)$ \\
\hline HTN & & & & $p=0.0006$ & & & & $p=0.4147$ \\
\hline without & $83.8 \%(848 / 1012)$ & $61.2 \%(311 / 508)$ & 旦 & $0.808(0.784-0.831)$ & $78.7 \%(288 / 366)$ & $46.5 \%(66 / 142)$ & He= & $0.675(0.624-0.727)$ \\
\hline with & $59.0 \%(196 / 332)$ & $75.7 \%(1091 / 1441)$ & 日 & $0.742(0.712-0.771)$ & $69.3 \%(731 / 1055)$ & $51.0 \%(197 / 386)$ & 日 & $0.650(0.619-0.682)$ \\
\hline HLP & & & & $p<0.0001$ & & & & $p=0.7211$ \\
\hline without & $82.1 \%(785 / 956)$ & $69.1 \%(394 / 570)$ & H & $0.846(0.826-0.865)$ & $72.0 \%(301 / 418)$ & $46.7 \%(71 / 152)$ & E-1 & $0.647(0.596-0.698)$ \\
\hline with & $66.8 \%(259 / 388)$ & $73.1 \%(1008 / 1379)$ & ㅂ & $0.762(0.735-0.789)$ & $71.6 \%(718 / 1003)$ & $51.1 \%(192 / 376)$ & E & $0.658(0.626-0.690)$ \\
\hline CKD & & & & $p=0.1188$ & & & & $p=0.1487$ \\
\hline without & $79.3 \%(1010 / 1274)$ & $63.9 \%(739 / 1157)$ & н & $0.798(0.781-0.816)$ & $77.7 \%(674 / 867)$ & $47.6 \%(138 / 290)$ & $\boxminus$ & $0.666(0.629-0.702)$ \\
\hline with & $48.6 \%(34 / 70)$ & $83.7 \%(663 / 792)$ & $\mapsto$ & $0.748(0.688-0.809)$ & $62.3 \%(345 / 554)$ & $52.5 \%(125 / 238)$ & $\boxminus$ & $0.625(0.584-0.666)$ \\
\hline STK & & & & $p=0.0008$ & & & & $p=0.3722$ \\
\hline without & $79.2 \%(1006 / 1271)$ & $70.9 \%(1129 / 1592)$ & н & $0.829(0.814-0.844)$ & $72.7 \%(839 / 1154)$ & $49.8 \%(218 / 438)$ & 目 & $0.661(0.632-0.691)$ \\
\hline with & $52.1 \%(38 / 73)$ & $76.5 \%(273 / 357)$ & $\mathrm{HEH}$ & $0.711(0.644-0.778)$ & $67.4 \%(180 / 267)$ & $50.0 \%(45 / 90)$ & H & $0.628(0.561-0.695)$ \\
\hline CAD & & & & $p=0.0254$ & & & & $p=0.6627$ \\
\hline without & $79.7 \%(921 / 1155)$ & $69.1 \%(746 / 1079)$ & н & $0.822(0.804-0.839)$ & $75.2 \%(594 / 790)$ & $48.1 \%(139 / 289)$ & 日 & $0.661(0.624-0.697)$ \\
\hline with & $65.1 \%(123 / 189)$ & $75.4 \%(656 / 870)$ & 日 & $0.776(0.740-0.812)$ & $67.4 \%(425 / 631)$ & $51.9 \%(124 / 239)$ & $\theta$ & $0.649(0.608-0.689)$ \\
\hline HF & & & & $p=0.1982$ & & & & $p=0.5057$ \\
\hline without & $78.3 \%(1030 / 1315)$ & $70.1 \%(1207 / 1722)$ & H & $0.821(0.806-0.836)$ & $72.7 \%(923 / 1270)$ & $48.7 \%(220 / 452)$ & $\mathrm{E}$ & $0.655(0.626-0.685)$ \\
\hline with & $48.3 \%(14 / 29)$ & $85.9 \%(195 / 227)$ & $\mapsto-1$ & $0.756(0.659-0.853)$ & $63.6 \%(96 / 151)$ & $56.6 \%(43 / 76)$ & 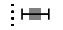 & $0.628(0.553-0.703)$ \\
\hline AF & & & & $p=0.1924$ & & & & $p=0.2430$ \\
\hline without & $78.2 \%(1039 / 1329)$ & $71.3 \%(1312 / 1839)$ & н & $0.824(0.810-0.839)$ & $71.5 \%(964 / 1349)$ & $51.2 \%(251 / 490)$ & 旦 & $0.659(0.631-0.687)$ \\
\hline with & $33.3 \%(5 / 15)$ & $81.8 \%(90 / 110)$ & $\longmapsto-1$ & $0.747(0.631-0.862)$ & $76.4 \%(55 / 72)$ & $31.6 \%(12 / 38)$ & $\because=-1$ & $0.591(0.480-0.701)$ \\
\hline COPD & & & & $p=0.8527$ & & & & $p=0.6848$ \\
\hline without & $78.2 \%(916 / 1171)$ & $71.4 \%(1090 / 1527)$ & H & $0.823(0.807-0.839)$ & $72.0 \%(796 / 1105)$ & $48.1 \%(203 / 422)$ & 日 & $0.654(0.623-0.684)$ \\
\hline with & $74.0 \%(128 / 173)$ & 73.9\% (312/422) & 日 & $0.827(0.790-0.864)$ & $70.6 \%(223 / 316)$ & $56.6 \%(60 / 106)$ & 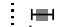 & $0.667(0.607-0.727)$ \\
\hline & & & & & & & 1 & \\
\hline
\end{tabular}

Figure 4. Stratified analysis for detecting DM and severe DM in the follow-up cohort. The DLM's sensitivity and specificity to detect DM and severe DM are tabulated across a series of stratified analyses. The $p$-value was the significant test of strength of association, and a significance level was 0.0045 based on the Bonferroni correction.

Figure $5 \mathrm{~A}$ shows that the higher ECG-HbA1c groups exhibit higher BMI, higher prevalence of $\mathrm{CKD} / \mathrm{HF} / \mathrm{HTN}$, worse kidney function (eGFR and blood urea nitrogen), lower Alb, and lower high-density lipoprotein cholesterol compared with the lower ECG-HbA1c groups, which are the risk factors for DM-related complications. Figure 5B shows outcome analysis of both DM/Lab-HbA1c and ECG-HbA1c. The false positive detection by DLM (ECG-HbA1c $\geq 6.5 \%$ ) shows higher HRs on three outcomes of interest compared to the true negative (ECG-HbA1c $<6.5 \%$ ) in patients without DM. Moreover, the false negative group (ECG-HbA1c $<6.5 \%$ ) presented the lower risk of these outcomes compared to true positives (ECG-HbA1c $\geq 6.5 \%$ ) in patients with DM. The dose response effects of ECG-HbA1c were significant on mortality (HR: 1.53, 95\% CI: 1.08-2.17), new-onset CKD (HR: 1.56, 95\% CI: 1.30-1.87), and new-onset HF (HR: 1.51, 95\% CI: 1.13-2.01) after gender and age adjustments, which was higher than the effects of Lab-HbA1c (HR of mortality: 0.95, 95\% CI: 0.73-1.24; HR of new-onset CKD: 1.24, 95\% CI: 1.07-1.43; HR of new-onset HF: 1.17, 95\% CI: 0.93-1.47). All the results demonstrated the beneficial role of ECG-HbA1c on the prediction of the cardiovascular disease outcomes compared to Lab-HbA1c. 
A
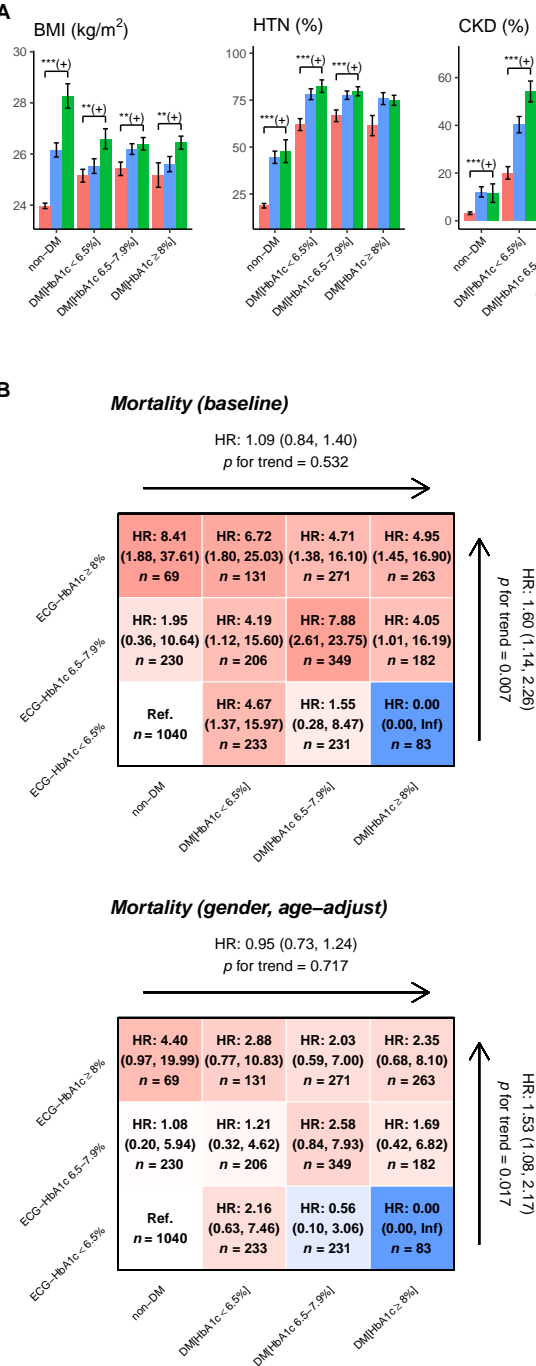
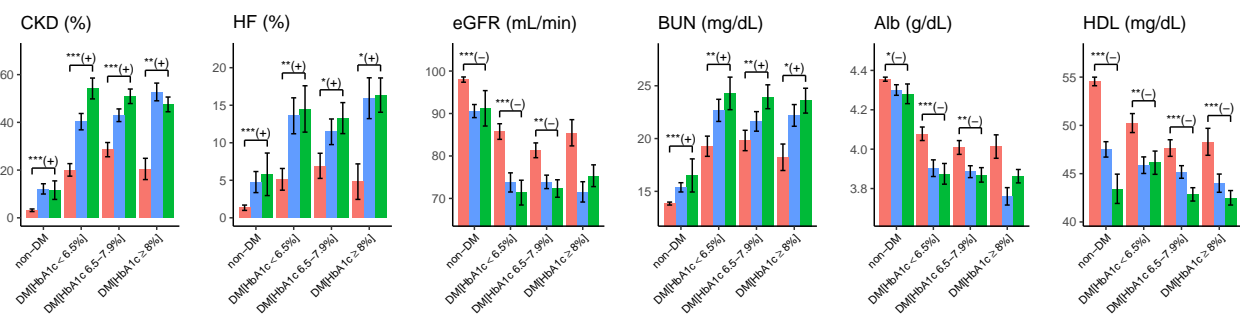

ECG-HbA1C $<6.5 \%$ ECG-HbA1c $6.5-7.9 \% \quad$ ECG-HbA1C $\geq 8 \%$
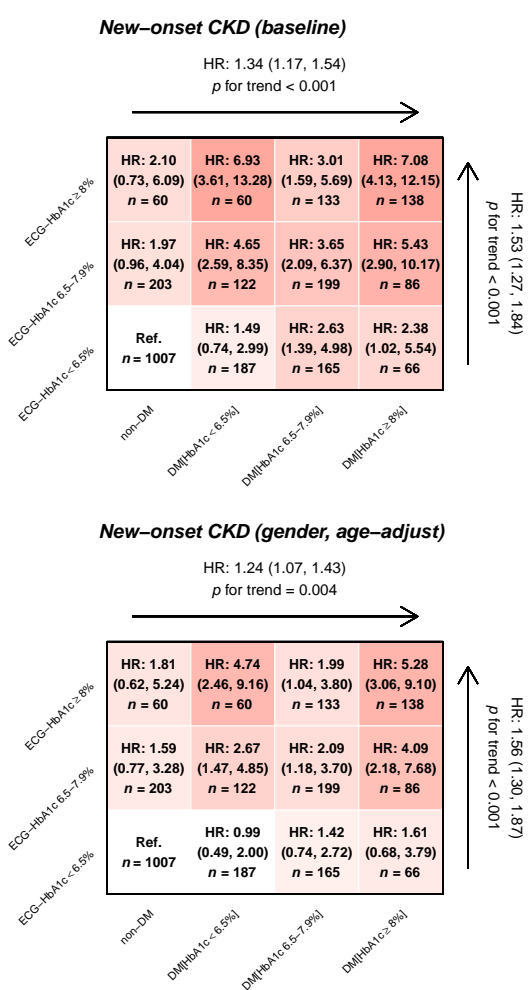
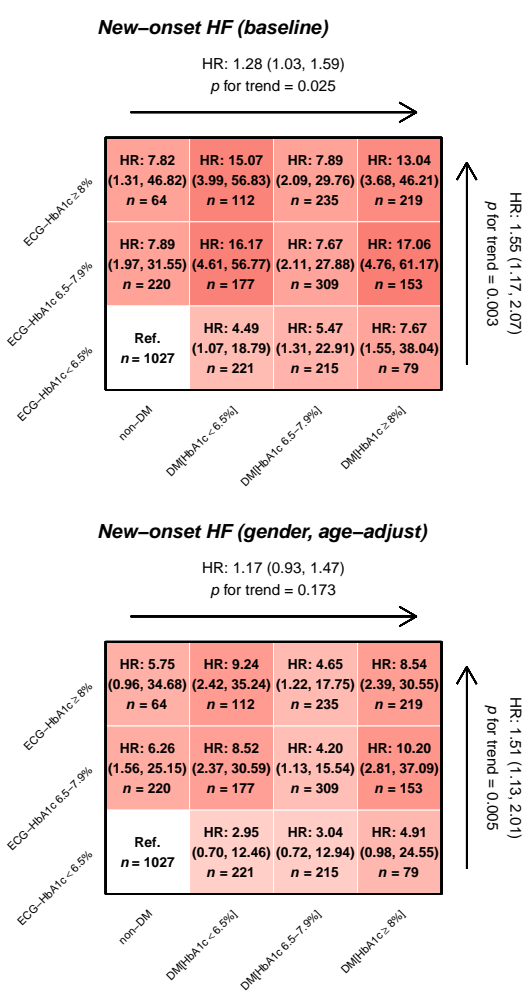

Figure 5. Characteristics and risk analysis in patients with actual HbA1c and ECG-HbA1c. (A) Patient characteristics in different ECG-HbA1c groups and real HbA1c groups. Bars represent the mean or proportion where appropriate and corresponding $95 \%$ conference intervals, which are adjusted by real $\mathrm{HbA} 1 \mathrm{c}$ in each group via linear or logistic regression. Significant tests are based on the trend test $\left({ }^{*}: p\right.$ for trend $<0.05 ;{ }^{* *}: p$ for trend $<0.01 ;{ }^{* * *}: p$ for trend $\left.<0.001\right)$, and the sign represents the correlation direction. (B) Risk matrixes of ECG-HbA1c and HbA1c groups on DM related complications. The hazard ratios (HRs) are based on a Cox proportional hazard model before and after adjusting by gender and age. The color gradient represents the risk of corresponding group.

Figure 6A shows additive effects of ECG-HbA1c. In the mortality analysis, the ECG-HbA1c provided a C-index of 0.665 (95\% CI: 0.600-0.730) which was significantly higher than Lab-HbA1c (C-index $=0.604,95 \%$ CI: 0.536-0.673). After full adjustments, ECG-HbA1c provided significantly more information on mortality (C-index $=0.835$ in model $3+$ ECGHbA1c) compared to Lab-HbA1c (C-index $=0.831$ in model $3+\mathrm{HbA1c}$ ), which is similar on the prediction of new-onset CKD. For the new-onset HF, the integration of Lab-HbA1c and ECG-HbA1c provided a higher C-index (0.665) compared to the Lab-HbA1c alone (0.620). In the full adjustment model including Lab-HbA1c, the integration of ECG-HbA1c significantly improved the model performance (C-index: 0.793 to $0.796, p<0.05$ ). Figure $6 \mathrm{~B}$ shows the HRs of the full adjustment model with Lab-HbA1c and ECG- HbA1c on these three outcomes. The ECG-HbA1c independently provided risk predictions (HR of mortality: 1.23, 95\% CI: 1.04-1.45; HR of new-onset CKD: 1.29, 95\% CI: 1.18-1.41; HR of new-onset HF: 1.20, 95\% CI: 1.03-1.39) in additional to a series of risk factors. Although the HRs of Lab-HbA1c were significant on the prediction of new-onset CKD (HR: 1.08, 95\% CI: 1.01-1.16) and new-onset 
HF (HR: 1.17, 95\% CI: 1.06-1.30), the effect of Lab-HbA1c was less than ECG-HbA1c. These results highlighted the strength of ECG-HbA1c to provide information on the unmeasured heart state.

A

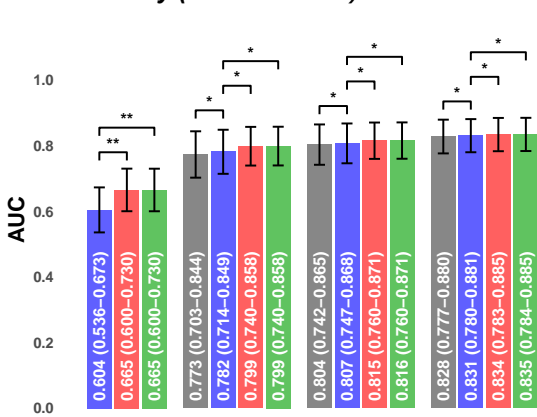

0.0
New-onset CKD (at risk $=2426$ )
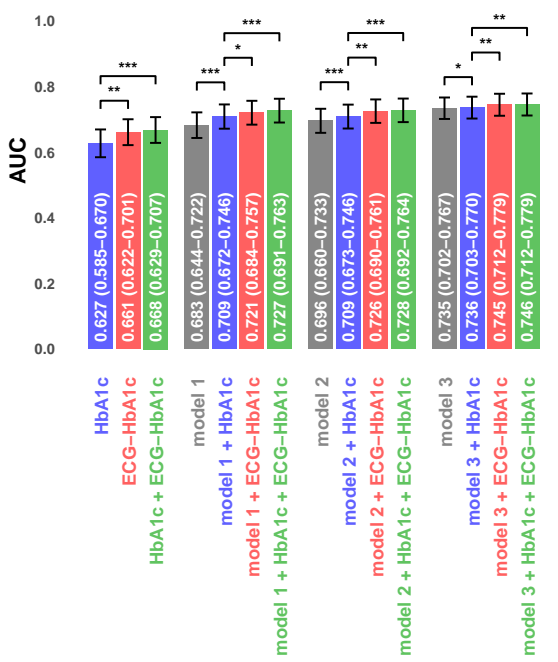

New-onset HF (at risk = 3031)

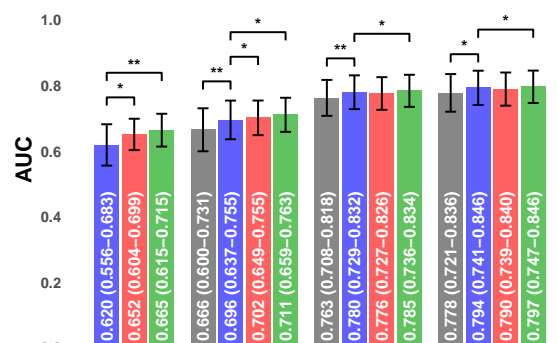

0.0

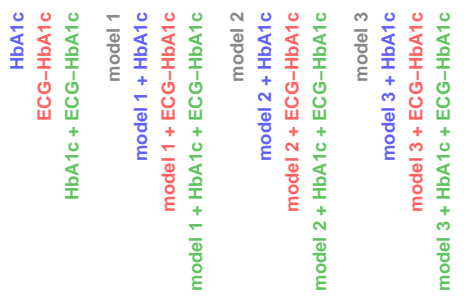

B
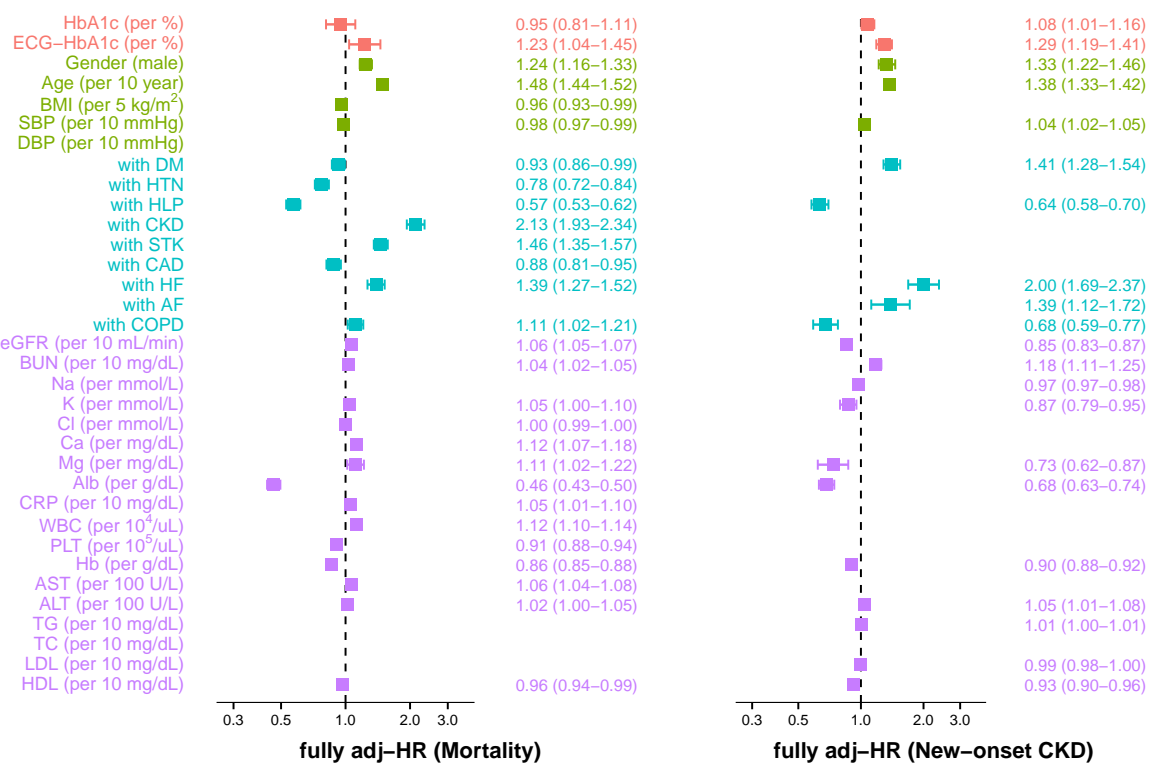

Figure 6. Additional contributions of ECG-HbA1c on DM related complications. (A) A Cox proportional hazard model and C-index are used as the performance assessment for a series of models. The model 1 includes significant demographic data, the model 2 includes variables in model 1 and additional significant disease histories, and the model 3 includes variables in model 2 and additional significant laboratory tests. Abbreviations: ${ }^{*}, p<0.05 ;{ }^{* *}, p<0.01 ;{ }^{* * *}, p<0.001$. (B) The multivariable analyses of the models with best performance (model $3+\mathrm{HbA} 1 \mathrm{c}+\mathrm{ECG}-\mathrm{HbA} 1 \mathrm{c}$ ) described above. The risk score can be calculated based on these coefficients to provide the corresponding C-index as above.

\section{Discussions}

Our ECG-HbA1c provides an AUC of 0.8255 on DM screening in follow-up cohorts. The underlying characteristic differences in different ECG-HbA1c under the same $\mathrm{Lab}-\mathrm{HbA} 1 \mathrm{c}$ were analyzed, which revealed patients with higher ECG-HbA1c had more risk factors for DM progression. ECG-HbA1c provides additional information, although we had already adjusted for full baseline characteristics. We believe that ECG furnishes 
more information on latent cardiovascular factors compared to Lab-HbA1c, especially in unmeasured factors.

Several ECG manifestations have been proposed as a means of determining diabetic disease status. Diabetic rats exhibited prolonged ventricular depolarization time, decreased conduction velocity, and increased arrhythmia during reperfusion, which are reflected in ECG [40]. In human studies, increased resting heart rate [41] and longer atrial conduction time [42] were found to be correlated with DM. Long-term impaired fasting glucose was also observed to lead to accelerated RHR, ST-T changes, and arrhythmias in ECG [43]. Our data demonstrates that heart rate, corrected QT interval, QT interval, and RS wave axis were the most important ECG changes in the prediction of Lab-HbA1c during big data analysis. However, the detection of DM by ECG is difficult. DLM has been found to extract features unrecognizable to humans, such as obtaining cardiovascular risk factors from the retinal fundus [44], contributing to better performance than that of XGB models and elastic nets.

The advantage of DLM compared to traditional methods is to extract useful features automatically [20]. Recently, a study developed a DLM for screening DM via ECG with AUCs of 0.777 in an OPD experiment [45]. Through the larger database and augmentation from GLU, our DLM achieved an AUC of 0.8255. Moreover, both previous [45] and our own studies show that ECG based DM detection is more accurate for people with normal ranges of weight. The MAE of our noninvasive system (1.238) even approximately reached the 13 commercially available point-of-care $\mathrm{HbA} 1 \mathrm{c}$ test devices ranging from -0.9 to 0.7 [46]. Importantly, our study further explores the meaning of predicting error, and finally points out the poor conditions in patients with higher ECG-HbA1c. ECG-HbA1c may be used to predict DM-related progression, which is critical in the identification of high-risk groups.

Although Lab-HbA1c may be the most important factor for prediction of DM progression [33], large amounts of missing data might preclude analysis by retrospective electronic medical records [16]. For example, cholesterol values were available for fewer than $30 \%$ of patients due to fewer measurements [47], necessitating a substitute, such as BMI, for the assessment of cardiovascular health [48,49]. Moreover, Lab-HbA1c might not be a perfect index for evaluating DM, especially in aged patients without DM [50,51]. Agedependent $\mathrm{HbA} 1 \mathrm{c}$ reference intervals for the diagnosis of $\mathrm{DM}$ have been proposed [52]. Our data demonstrated that ECG-HbA1c might be feasible when missing Lab-HbA1c values, and even has a higher predictive ability in regard to mortality, new-onset CKD and HF compared to Lab-HbA1c. Moreover, our data demonstrates that patients with higher ECG-HbA1c under the same laboratory-based $\mathrm{HbA1c}$ present increased risk factors for DM progression, indicating that ECG-HbA1c provides additional predictive information, even when the Lab-HbA1c is available. Similarly, it had been suggested that patients with higher ECG age under the same chronological age usually have a higher incidence of hypertension, CAD, or low ejection fractions [32]. Patients with abnormal ECG-based ejection fractions also exhibit a fourfold increased risk for developing future ventricular dysfunction [27]. Taken together, these results emphasize the beneficial effects of an ECG-based system for screening DM and predicting its progression, which warrants further validation in large-scale community studies.

The strength of our study is in conducting a series of experiments to apply different training strategies involving epidemiological perspectives and the gender/age-matching strategy with simulated $\mathrm{HbA1c}$ based on GLU to demonstrate the best performance. The matching strategy avoids the DLM learning spurious correlations, which maximizes additional notable ECG features. A previous study demonstrated the superiority of a matching strategy that avoids identifying discharge notes of neoplasms using negative terms, such as pregnancy [53]. Although matching strategies may not substantially increase DLM performance, they learn causality, which improves extrapolation. Gender and age are related to DM [54,55], and previous studies have shown correlations among gender, age, and ECG [32]. These relationships have led us to consider the possibility of confounding effects whenever these factors causally influence both ECG and HbA1c [56]. The gender/age- 
matching strategy not only provides a higher correlation, but also shows a lower correlation with age and gender. To the best of our knowledge, there is no DLM research that considers these potential confounding effects. Future medical DLM research may need to further analyze the source of predictive power and try to use matching strategies to improve learning quality.

Some limitations of this study should be acknowledged. First, this is a hospital-based retrospective study. Our data indicate the improved value of ECG-HbA1c, and we consider a community-based prospective study necessary to validate the effect of ECG-HbA1c. Second, ECG characteristics may vary by race, although the diagnostic performance of DLM may be still stable [57]. An international study involving different racial and ethnic groups should still be conducted to validate the advantage of ECG-HbA1c. Third, DM can be classified into 4 types, including type 1 diabetes, type 2 diabetes, gestational diabetes mellitus, and specific types of diabetes due to other causes [35]. Although type 2 diabetes is the most predominant type in the study, we could not provide the detailed type of diabetes of each patient. Finally, the "black box" of DLM necessitates our ECG-HbA1c being more transparent [58]. Although traditional explainable models reveal some clues, their performances are significantly worse than that of DLM. Further studies should explore the relationship between ECG morphological findings and DM severities.

\section{Conclusions}

In this study, we developed a novel biomarker, ECG-HbA1c, for predicting the risks and progression of DM and its related complications. In addition to clinical practice, our study creates a new avenue for using matching strategies for training DLMs, which avoids learning spurious correlations. Moreover, ECG is a simple, inexpensive, and noninvasive test that is suitable for applications in large-scale community settings. ECG-HbA1c is not only considered as a tool for initial DM screening, but also provides additional information on DM progression, even with available laboratory data. Although further studies are necessary, this system provides promising ECG-based indicators to promote health care quality in patients with DM.

Author Contributions: All authors participated in designing the study, generating hypotheses, interpreting the data, and critically reviewing the paper. C.-S.L. and C.L. wrote the first draft, and Y.-T.L. and F.-C.K. contributed substantially to the writing of subsequent versions. Statistical analyses were designed and conducted by Y.-S.L. with support from W.-H.F., C.-C.L. and C.L. All authors had full access to all the data in the study and accept responsibility for the decision to submit for publication. C.-S.L. and C.L. verified all the data used in this study. The corresponding author (C.L.) attests that all listed authors meet authorship criteria and that no others meeting the criteria have been omitted. C.L. had final responsibility for the decision to submit for publication. All authors have read and agreed to the published version of the manuscript.

Funding: This study was supported by funding from the Ministry of Science and Technology, Taiwan (MOST 108-2314-B-016-001 to C. Lin, MOST 109-2314-B-016-026 to C. Lin, MOST110-2314-B-016-010MY3 to C. Lin, and MOST 106-2314-B-016-038-MY3 to C.S. Lin), the Tri-Service General Hospital, Taiwan (TSGH-C107-007-007-S02 to C.S. Lin), the National Science and Technology Development Fund Management Association, Taiwan (MOST 108-3111-Y-016-009 and MOST 109-3111-Y-016-002 to C. Lin), and the Cheng Hsin General Hospital, Taiwan (CHNDMC-109-19 to C. Lin and CHNDMC-110-15 to C. Lin).

Institutional Review Board Statement: This study was approved by the institutional review board in Tri-Service General Hospital, Taipei, Taiwan (IRB NO. C202105049).

Informed Consent Statement: Patients' consent was waived because data were collected retrospectively and in anonymized files and encrypted from the hospital to the data controller.

Data Availability Statement: The data presented in this study are available on request from the corresponding author.

Conflicts of Interest: The authors declare no conflict of interest. 


\section{References}

1. Saeedi, P.; Petersohn, I.; Salpea, P.; Malanda, B.; Karuranga, S.; Unwin, N.; Colagiuri, S.; Guariguata, L.; Motala, A.A.; Ogurtsova, K.; et al. Global and regional diabetes prevalence estimates for 2019 and projections for 2030 and 2045: Results from the international diabetes federation diabetes atlas, 9th edition. Diabetes Res. Clin. Pract. 2019, 157, 107843. [CrossRef]

2. Chatterjee, S.; Khunti, K.; Davies, M.J. Type 2 diabetes. Lancet 2017, 389, 2239-2251. [CrossRef]

3. John, W.G.; Hillson, R.; Alberti, S.G. Use of haemoglobin A1c (HbA1c) in the diagnosis of diabetes mellitus. The implementation of World Health Organisation (WHO) guidance 2011. Pract. Diabetes 2012, 29, 12-12a. [CrossRef]

4. Lindström, J.; Tuomilehto, J. The diabetes risk score: A practical tool to predict type 2 diabetes risk. Diabetes Care 2003, 26, 725-731. [CrossRef] [PubMed]

5. Rahman, M.; Simmons, R.K.; Harding, A.H.; Wareham, N.J.; Griffin, S.J. A simple risk score identifies individuals at high risk of developing Type 2 diabetes: A prospective cohort study. Fam. Pract. 2008, 25, 191-196. [CrossRef]

6. Hippisley-Cox, J.; Coupland, C.; Robson, J.; Sheikh, A.; Brindle, P. Predicting risk of type 2 diabetes in England and Wales: Prospective derivation and validation of QDScore. BMJ 2009, 338, b880. [CrossRef]

7. Noble, D.; Mathur, R.; Dent, T.; Meads, C.; Greenhalgh, T. Risk models and scores for type 2 diabetes: Systematic review. BMJ 2011, 343, d7163. [CrossRef] [PubMed]

8. Long, A.N.; Dagogo-Jack, S. Comorbidities of diabetes and hypertension: Mechanisms and approach to target organ protection. J. Clin. Hypertens. 2011, 13, 244-251. [CrossRef] [PubMed]

9. Jelinek, H.F.; Osman, W.M.; Khandoker, A.H.; Khalaf, K.; Lee, S.; Almahmeed, W.; Alsafar, H.S. Clinical profiles, comorbidities and complications of type 2 diabetes mellitus in patients from United Arab Emirates. BMJ Open Diabetes Res. Care 2017, 5, e000427. [CrossRef]

10. Htay, T.; Soe, K.; Lopez-Perez, A.; Doan, A.H.; Romagosa, M.A.; Aung, K. Mortality and Cardiovascular Disease in Type 1 and Type 2 Diabetes. Curr. Cardiol. Rep. 2019, 21, 45. [CrossRef] [PubMed]

11. Standards of medical care in diabetes-2015 abridged for primary care providers. Clin. Diabetes Publ. Am. Diabetes Assoc. 2015, 33, 97-111. [CrossRef]

12. Sabanayagam, C.; Liew, G.; Tai, E.S.; Shankar, A.; Lim, S.C.; Subramaniam, T.; Wong, T.Y. Relationship between glycated haemoglobin and microvascular complications: Is there a natural cut-off point for the diagnosis of diabetes? Diabetologia 2009, 52, 1279-1289. [CrossRef]

13. El-Salem, K.; Ammari, F.; Khader, Y.; Dhaimat, O. Elevated glycosylated hemoglobin is associated with subclinical neuropathy in neurologically asymptomatic diabetic patients: A prospective study. J. Clin. Neurophysiol. 2009, 26, 50-53. [CrossRef]

14. Vos, F.E.; Schollum, J.B.; Walker, R.J. Glycated albumin is the preferred marker for assessing glycaemic control in advanced chronic kidney disease. NDT Plus 2011, 4, 368-375. [CrossRef] [PubMed]

15. Khaw, K.T.; Wareham, N.; Bingham, S.; Luben, R.; Welch, A.; Day, N. Association of hemoglobin A1c with cardiovascular disease and mortality in adults: The European prospective investigation into cancer in Norfolk. Ann. Intern. Med. 2004, 141, 413-420. [CrossRef]

16. Chowdhury, M.; Goonan, K.; Peacock, S.; Simpson, R. On Missing Values of HbA1c in Diabetes Quality of Care Evaluation. Diabetes 2001, 50 (Suppl. 2).

17. Murdoch, T.B.; Detsky, A.S. The inevitable application of big data to health care. JAMA 2013, 309, 1351-1352. [CrossRef]

18. Yeboah, J.; McClelland, R.L.; Polonsky, T.S.; Burke, G.L.; Sibley, C.T.; O'Leary, D.; Carr, J.J.; Goff, D.C.; Greenland, P.; Herrington, D.M. Comparison of novel risk markers for improvement in cardiovascular risk assessment in intermediate-risk individuals. JAMA 2012, 308, 788-795. [CrossRef] [PubMed]

19. Diller, G.P.; Kempny, A.; Babu-Narayan, S.V.; Henrichs, M.; Brida, M.; Uebing, A.; Lammers, A.E.; Baumgartner, H.; Li, W.; Wort, S.J.; et al. Machine learning algorithms estimating prognosis and guiding therapy in adult congenital heart disease: Data from a single tertiary centre including 10,019 patients. Eur. Heart J. 2019, 40, 1069-1077. [CrossRef] [PubMed]

20. LeCun, Y.; Bengio, Y.; Hinton, G. Deep learning. Nature 2015, 521, 436-444. [CrossRef] [PubMed]

21. Liu, W.C.; Lin, C.S.; Tsai, C.S.; Tsao, T.P.; Cheng, C.C.; Liou, J.T.; Lin, W.S.; Cheng, S.M.; Lou, Y.S.; Lee, C.C.; et al. A Deep-Learning Algorithm for Detecting Acute Myocardial Infarction. EuroIntervention J. EuroPCR Collab. Work. Group Interv. Cardiol. Eur. Soc. Cardiol. 2021. [CrossRef]

22. Chang, D.-W.; Lin, C.-S.; Tsao, T.-P.; Lee, C.-C.; Chen, J.-T.; Tsai, C.-S.; Lin, W.-S.; Lin, C. Detecting Digoxin Toxicity by Artificial Intelligence-Assisted Electrocardiography. Int. J. Environ. Res. Public Health 2021, 18, 3839. [CrossRef] [PubMed]

23. Attia, Z.I.; Noseworthy, P.A.; Lopez-Jimenez, F.; Asirvatham, S.J.; Deshmukh, A.J.; Gersh, B.J.; Carter, R.E.; Yao, X.; Rabinstein, A.A.; Erickson, B.J.; et al. An artificial intelligence-enabled ECG algorithm for the identification of patients with atrial fibrillation during sinus rhythm: A retrospective analysis of outcome prediction. Lancet 2019, 394, 861-867. [CrossRef]

24. Hannun, A.Y.; Rajpurkar, P.; Haghpanahi, M.; Tison, G.H.; Bourn, C.; Turakhia, M.P.; Ng, A.Y. Cardiologist-level arrhythmia detection and classification in ambulatory electrocardiograms using a deep neural network. Nat. Med. 2019, 25, 65-69. [CrossRef]

25. Galloway, C.D.; Valys, A.V.; Shreibati, J.B.; Treiman, D.L.; Petterson, F.L.; Gundotra, V.P.; Albert, D.E.; Attia, Z.I.; Carter, R.E.; Asirvatham, S.J.; et al. Development and validation of a deep-learning model to screen for hyperkalemia from the electrocardiogram. JAMA Cardiol. 2019, 4, 428-436. [CrossRef] [PubMed] 
26. Lin, C.S.; Lin, C.; Fang, W.H.; Hsu, C.J.; Chen, S.J.; Huang, K.H.; Lin, W.S.; Tsai, C.S.; Kuo, C.C.; Chau, T.; et al. A deep-learning algorithm (ECG12Net) for detecting hypokalemia and hyperkalemia by electrocardiography: Algorithm development. JMIR Med. Inform. 2020, 8, e15931. [CrossRef] [PubMed]

27. Attia, Z.I.; Kapa, S.; Lopez-Jimenez, F.; McKie, P.M.; Ladewig, D.J.; Satam, G.; Pellikka, P.A.; Enriquez-Sarano, M.; Noseworthy, P.A.; Munger, T.M.; et al. Screening for cardiac contractile dysfunction using an artificial intelligence-enabled electrocardiogram. Nat. Med. 2019, 25, 70-74. [CrossRef] [PubMed]

28. Attia, Z.I.; Kapa, S.; Yao, X.; Lopez-Jimenez, F.; Mohan, T.L.; Pellikka, P.A.; Carter, R.E.; Shah, N.D.; Friedman, P.A.; Noseworthy, P.A. Prospective validation of a deep learning electrocardiogram algorithm for the detection of left ventricular systolic dysfunction. J. Cardiovasc. Electrophysiol. 2019, 30, 668-674. [CrossRef]

29. Kwon, J.M.; Kim, K.H.; Akkus, Z.; Jeon, K.H.; Park, J.; Oh, B.H. Artificial intelligence for detecting mitral regurgitation using electrocardiography. J. Electrocardiol. 2020, 59, 151-157. [CrossRef]

30. Kwon, J.M.; Lee, S.Y.; Jeon, K.H.; Lee, Y.; Kim, K.H.; Park, J.; Oh, B.H.; Lee, M.M. Deep learning-based algorithm for detecting aortic stenosis using electrocardiography. J. Am. Heart Assoc. 2020, 9, e014717. [CrossRef]

31. Porumb, M.; Stranges, S.; Pescape, A.; Pecchia, L. Precision medicine and artificial intelligence: A pilot study on deep learning for hypoglycemic events detection based on ECG. Sci. Rep. 2020, 10, 170. [CrossRef] [PubMed]

32. Attia, Z.I.; Friedman, P.A.; Noseworthy, P.A.; Lopez-Jimenez, F.; Ladewig, D.J.; Satam, G.; Pellikka, P.A.; Munger, T.M.; Asirvatham, S.J.; Scott, C.G.; et al. Age and sex estimation using artificial intelligence from standard 12-lead ECGs. Circ. Arrhythmia Electrophysiol. 2019, 12, e007284. [CrossRef]

33. Nakagami, T.; Tajima, N.; Oizumi, T.; Karasawa, S.; Wada, K.; Kameda, W.; Susa, S.; Kato, T.; Daimon, M. Hemoglobin A1c in predicting progression to diabetes. Diabetes Res. Clin. Pract. 2010, 87, 126-131. [CrossRef]

34. Buuren, S.v.; Groothuis-Oudshoorn, K. Mice: Multivariate imputation by chained equations in R. J. Stat. Softw. 2010, 45, 1-67. [CrossRef]

35. American Diabetes Association. 2. Classification and Diagnosis of Diabetes: Standards of Medical Care in Diabetes-2021. Diabetes Care 2021, 44, S15-S33. [CrossRef] [PubMed]

36. American Diabetes Association. 6. Glycemic Targets: Standards of Medical Care in Diabetes-2018. Diabetes Care 2018, 41, S55-S64. [CrossRef] [PubMed]

37. Lin, C.; Lin, C.-S.; Lee, D.-J.; Lee, C.-C.; Chen, S.-J.; Tsai, S.-H.; Kuo, F.-C.; Chau, T.; Lin, S.-H. Artificial intelligence assisted electrocardiography for early diagnosis of thyrotoxic periodic paralysis. J. Endocr. Soc. 2021, 5, bvab120. [CrossRef]

38. Lin, C.; Hsu, C.J.; Lou, Y.S.; Yeh, S.J.; Lee, C.C.; Su, S.L.; Chen, H.C. Artificial Intelligence Learning Semantics via External Resources for Classifying Diagnosis Codes in Discharge Notes. J. Med. Internet Res. 2017, 19, e380. [CrossRef]

39. Simpson, A.J. Over-sampling in a deep neural network. arXiv 2015, arXiv:1502.03648.

40. Axelsen, L.N.; Calloe, K.; Braunstein, T.H.; Riemann, M.; Hofgaard, J.P.; Liang, B.; Jensen, C.F.; Olsen, K.B.; Bartels, E.D.; Baandrup, U.; et al. Diet-induced pre-diabetes slows cardiac conductance and promotes arrhythmogenesis. Cardiovasc. Diabetol. 2015, 14, 87. [CrossRef]

41. Yang, Z.; Zhang, W.; Zhu, L.; Lin, N.; Niu, Y.; Li, X.; Lu, S.; Zhang, H.; Wang, X.; Wen, J.; et al. Resting heart rate and impaired glucose regulation in middle-aged and elderly Chinese people: A cross-sectional analysis. BMC Cardiovasc. Disord. 2017, 17, 246. [CrossRef]

42. Gudul, N.E.; Karabag, T.; Sayin, M.R.; Bayraktaroglu, T.; Aydin, M. Atrial conduction times and left atrial mechanical functions and their relation with diastolic function in prediabetic patients. Korean J. Intern. Med. 2017, 32, 286-294. [CrossRef]

43. Wang, L.; Cui, L.; Wang, Y.; Vaidya, A.; Chen, S.; Zhang, C.; Zhu, Y.; Li, D.; Hu, F.B.; Wu, S.; et al. Resting heart rate and the risk of developing impaired fasting glucose and diabetes: The Kailuan prospective study. Int. J. Epidemiol. 2015, 44, 689-699. [CrossRef] [PubMed]

44. Poplin, R.; Varadarajan, A.V.; Blumer, K.; Liu, Y.; McConnell, M.V.; Corrado, G.S.; Peng, L.; Webster, D.R. Prediction of cardiovascular risk factors from retinal fundus photographs via deep learning. Nat. Biomed. Eng. 2018, 2, 158-164. [CrossRef] [PubMed]

45. Wang, L.; Mu, Y.; Zhao, J.; Wang, X.; Che, H. IGRNet: A Deep Learning Model for Non-Invasive, Real-Time Diagnosis of Prediabetes through Electrocardiograms. Sensors 2020, 20, 2556. [CrossRef]

46. Hirst, J.A.; McLellan, J.H.; Price, C.P.; English, E.; Feakins, B.G.; Stevens, R.J.; Farmer, A.J. Performance of point-of-care HbA1c test devices: Implications for use in clinical practice-A systematic review and meta-analysis. Clin. Chem. Lab. Med. 2017, 55, 167-180. [CrossRef] [PubMed]

47. Hira, R.S.; Kennedy, K.; Nambi, V.; Jneid, H.; Alam, M.; Basra, S.S.; Ho, P.M.; Deswal, A.; Ballantyne, C.M.; Petersen, L.A.; et al. Frequency and practice-level variation in inappropriate aspirin use for the primary prevention of cardiovascular disease: Insights from the National Cardiovascular Disease Registry's Practice Innovation and Clinical Excellence registry. J. Am. Coll. Cardiol. 2015, 65, 111-121. [CrossRef]

48. Cooney, M.T.; Dudina, A.; De Bacquer, D.; Fitzgerald, A.; Conroy, R.; Sans, S.; Menotti, A.; De Backer, G.; Jousilahti, P.; Keil, U.; et al. How much does HDL cholesterol add to risk estimation? A report from the SCORE Investigators. Eur. J. Cardiovasc. Prev. Rehabil. 2009, 16, 304-314. [CrossRef] 
49. Dudina, A.; Cooney, M.T.; Bacquer, D.D.; Backer, G.D.; Ducimetière, P.; Jousilahti, P.; Keil, U.; Menotti, A.; Njølstad, I.; Oganov, R.; et al. Relationships between body mass index, cardiovascular mortality, and risk factors: A report from the SCORE investigators. Eur. J. Cardiovasc. Prev. Rehabil. 2011, 18, 731-742. [CrossRef]

50. Pani, L.N.; Korenda, L.; Meigs, J.B.; Driver, C.; Chamany, S.; Fox, C.S.; Sullivan, L.; D’Agostino, R.B.; Nathan, D.M. Effect of aging on A1C levels in individuals without diabetes: Evidence from the Framingham Offspring Study and the National Health and Nutrition Examination Survey 2001-2004. Diabetes Care 2008, 31, 1991-1996. [CrossRef]

51. Dubowitz, N.; Xue, W.; Long, Q.; Ownby, J.G.; Olson, D.E.; Barb, D.; Rhee, M.K.; Mohan, A.V.; Watson-Williams, P.I.; Jackson, S.L.; et al. Aging is associated with increased HbA1c levels, independently of glucose levels and insulin resistance, and also with decreased HbA1c diagnostic specificity. Diabet. Med. 2014, 31, 927-935. [CrossRef]

52. Masuch, A.; Friedrich, N.; Roth, J.; Nauck, M.; Müller, U.A.; Petersmann, A. Preventing misdiagnosis of diabetes in the elderly: Age-dependent $\mathrm{HbA1c}$ reference intervals derived from two population-based study cohorts. BMC Endocr. Disord. $2019,19,20$. [CrossRef]

53. Lin, C.; Lou, Y.S.; Tsai, D.J.; Lee, C.C.; Hsu, C.J.; Wu, D.C.; Wang, M.C.; Fang, W.H. Projection Word Embedding Model with Hybrid Sampling Training for Classifying ICD-10-CM Codes: Longitudinal Observational Study. JMIR Med. Inform. 2019, 7, e14499. [CrossRef] [PubMed]

54. Legato, M.J.; Gelzer, A.; Goland, R.; Ebner, S.A.; Rajan, S.; Villagra, V.; Kosowski, M. Gender-specific care of the patient with diabetes: Review and recommendations. Gend. Med. 2006, 3, 131-158. [CrossRef]

55. Dong, W.; Wan, E.Y.F.; Bedford, L.E.; Wu, T.; Wong, C.K.H.; Tang, E.H.M.; Lam, C.L.K. Prediction models for the risk of cardiovascular diseases in Chinese patients with type 2 diabetes mellitus: A systematic review. Public Health 2020, 186, 144-156. [CrossRef] [PubMed]

56. VanderWeele, T.J.; Shpitser, I. On the definition of a confounder. Ann. Stat. 2013, 41, 196-220. [CrossRef] [PubMed]

57. Noseworthy, P.A.; Attia, Z.I.; Brewer, L.C.; Hayes, S.N.; Yao, X.; Kapa, S.; Friedman, P.A.; Lopez-Jimenez, F. Assessing and mitigating bias in medical artificial intelligence: The effects of race and ethnicity on a deep learning model for ECG analysis. Circ. Arrhythmia Electrophysiol. 2020, 13, e007988. [CrossRef]

58. Castelvecchi, D. Can we open the black box of AI? Nature 2016, 538, 20-23. [CrossRef] 